\title{
Automaticity of phasic alertness: Evidence for a three-component model of visual cueing
}

\author{
Zhicheng $\operatorname{Lin}^{1} \cdot$ Zhong-Lin Lu ${ }^{1}$
}

Published online: 12 May 2016

(C) The Psychonomic Society, Inc. 2016

\begin{abstract}
The automaticity of phasic alertness is investigated using the attention network test. Results show that the cueing effect from the alerting cue-double cue-is strongly enhanced by the task relevance of visual cues, as determined by the informativeness of the orienting cue - single cue - that is being mixed ( $80 \%$ vs. $50 \%$ valid in predicting where the target will appear). Counterintuitively, the cueing effect from the alerting cue can be negatively affected by its visibility, such that masking the cue from awareness can reveal a cueing effect that is otherwise absent when the cue is visible. Evidently, then, top-down influences - in the form of contextual relevance and cue awareness - can have opposite influences on the cueing effect from the alerting cue. These findings lead us to the view that a visual cue can engage three components of attention - orienting, alerting, and inhibition - to determine the behavioral cueing effect. We propose that phasic alertness, particularly in the form of specific response readiness, is regulated by both internal, top-down expectation and external, bottom-up stimulus properties. In contrast to some existing views, we advance the perspective that phasic alertness is strongly tied to temporal orienting, attentional capture, and spatial orienting. Finally, we discuss how translating attention research to clinical applications would benefit from an improved ability to measure attention. To this
\end{abstract}

Electronic supplementary material The online version of this article (doi:10.3758/s13414-016-1124-5) contains supplementary material, which is available to authorized users.

Zhicheng Lin

zhichenglin@gmail.com

1 Department of Psychology, The Ohio State University, 225 Psychology Building, 1835 Neil Avenue, Columbus, OH 43210, USA end, controlling the degree of intraindividual variability in the attentional components and improving the precision of the measurement tools may prove vital.

Keywords Phasic alertness · Exogenous cueing · Unconscious attention $\cdot$ Inhibition $\cdot$ Attention network test

During waking hours, our state of wakefulness is in flux, with attention and consciousness waxing and waning. A major source of this fluctuation is phasic alertness (Moruzzi \& Magoun, 1949). A change in the traffic light color, a knock on the door, a sudden touch on the shoulder - these salient events can momentarily heighten our response readiness. Phasic alertness has been conceptualized as a temporal attentional filter, to allow the organism to rapidly mobilize resources when they are most needed (Aston-Jones \& Cohen, 2005). This conceptualization raises a fundamental question: what gates the attentional filter of phasic alerting and determines its behavioral manifestation? In contrast to the intuitive and prevailing idea that stimulus salience is the key, here we report novel behavioral evidence that being salient is not sufficient for a task-irrelevant cue to alert attention, and nor is it necessary, which leads to a new perspective on the automaticity of phasic alerting and on the mechanisms of visual cueing in general.

The classic method for studying the behavioral footprints of phasic alerting is the attention network test (ANT). This procedure combines attentional cueing and response competition to isolate the alerting effect from spatial orienting and executive attention (Fan, McCandliss, Sommer, Raz, \& Posner, 2002). A key element in the procedure is the introduction of various warning cues before the target appears. When the cue appears at the two potential target locations, performance in this double cue condition is better than when there is 
no cue, revealing the alerting effect, which is thought to reflect the attentional benefit of monitoring over two target locations (Posner, 2008). Likewise, performance is better when a single cue appears at the same location as the target (valid) than at a different location (invalid), reflecting the orienting effect. On the other hand, manipulating the target display_-by flanking the target (e.g., a left-pointing arrow) with either a responsecompatible flanker (i.e., also a left-pointing arrow) or a response-incompatible flanker (i.e., a right-pointing arrow) - offers a probe into executive attention, as indexed by the interference effect. Here our focus is on orienting and alerting, which are dissociable from executive attention.

The ANT has been widely used in both basic and clinical research on attention, but how automatic phasic alertness is remains poorly understood. This is partly due to a subtle but critical methodological limitation in previous research. To facilitate voluntary orienting, many studies have examined orienting and alerting by using informative rather than completely uninformative single cues - as evidenced by there being more valid than invalid trials (Fan et al., 2009; Fan, McCandliss, Fossella, Flombaum, \& Posner, 2005; Fan et al., 2002). As a result, the double cue, although by its very nature uninformative of the target location, also may become task-relevant due to the incentive to attend to the cue - the single cue after all is informative (for demonstration of contextual influence on attention and perception, see $\mathrm{Lin}, \mathrm{Lu}, \&$ He, 2016; Lin \& Murray, 2014, 2015b). The potential influence of task relevance makes it difficult to ascertain whether the alerting effect obtained in the ANT is contingent on the double cue being mixed with informative single cues or it reflects stimulus-driven, bottom-up processes.

Here we probe the automaticity of phasic alerting in two ways. In Experiment 1, we directly manipulated the spatial informativeness of the single cue that was mixed with the double cue, and examined its influence on alerting across multiple intervals, as specified by the cue-target onset asynchrony (CTOA). In Experiment 2, we further tested how cue visibility modulates alerting. To preview the results, Experiment 1 showed that, compared with uninformative single cues, informative single cues strongly enhanced the alerting effect from the double cue. Experiment 2 further documented a counter-intuitive observation: a salient double cue failed to alert attention when the CTOA was very short and the mixed single cue was uninformative, but under the same limiting condition, the double cue could alert attention when it was rendered invisible through backward masking. Awareness, in other words, can come with a cost, in accordance with a recently documented phenomenon called awareness-dependent inhibition (Lin \& Murray, 2015a)-a phenomenon directly confirmed in the current data. The inhibition arises because when a salient cue is spatially and temporally close to the target but uninformative for the task, being wary of its potential distraction triggers the cognitive control system to inhibit the cue. These results provide novel evidence that being salient is neither sufficient nor necessary for a task-irrelevant cue to induce phasic alerting. Taken together, these findings suggest that top-down influences, in the form of contextual relevance and cue awareness, can have opposite influences on the alerting effect from the double cue.

\section{Experiment 1}

To study how the informativeness of the mixed single cue affects alerting, we used peripheral, abrupt-onset cues known to drive bottom-up attention (Yantis \& Jonides, 1990), as in prior research (Fan et al., 2002). Critically we manipulated the spatial informativeness of the single cue - known as cue validity or cue informativeness ${ }^{1}$ - by having more valid-cue trials than invalid-cue trials in the informative-cue group (as in some prior ANT studies), but equal proportions of the two in the uninformative-cue group. To track the temporal dynamics of cueing effects, we further randomized CTOAs - either $16.7 \mathrm{~ms}, 316.7 \mathrm{~ms}$, or $616.7 \mathrm{~ms}$ - within blocks (Fuentes \& Campoy, 2008; Posner \& Boies, 1971). These manipulations allowed us to assess the influence of context-based task relevance and CTOA on alerting and orienting. Given our focus on alerting and orienting, we used a simple dot detection task (Lin \& Murray, 2015a) instead of a flanker discrimination task (Fan et al., 2002).

The three CTOAs- $16.7 \mathrm{~ms}, 316.7 \mathrm{~ms}$, and $616.7 \mathrm{~ms}-$ were chosen based on two considerations. First, attentional facilitation from exogenous cues has been found to peak around $100 \mathrm{~ms}$ after the cue onset (Nakayama \& Mackeben, 1989), and the short CTOA of $16.7 \mathrm{~ms}$ allowed us to identify a potential limiting condition for exogenous cueing (in particular, for alerting). Second, endogenous facilitation from informative cues typically requires some time to manifest - e.g., $300 \mathrm{~ms}$ (Muller \& Rabbitt, 1989) - and the longer CTOAs of $316.7 \mathrm{~ms}$ and $616.7 \mathrm{~ms}$ allowed us to examine the role of topdown expectation in modulating alertness. The longer durations also provided us a chance to observe attentional inhibition from attentional capture in orienting (Klein, 2000).

\section{Method}

Participants Forty participants with normal or corrected-tonormal vision were recruited. They were split into two groups: the uninformative-cue group $(n=20 ; 12$ females, 8 males;

\footnotetext{
${ }^{1}$ We decide on cue informativeness rather than cue validity to refer to the spatial informativeness of the single cue. The primary reason is to avoid the potential confusion with subsequent data analysis, where validity is used as a factor to denote valid versus invalid trials. It also helps to avoid another potential confusion with category perception and learning, where cue validity is used to refer to the conditional probability that an object with a certain cue (feature) belongs to a particular category.
} 
mean age $=19.0$ years $)$ and the informative-cue group $(n=20$; 13 females, 7 males; mean age $=20.2$ years). The sample size of 20 - for this and the next experiment - was predetermined on the basis of recent studies using a similar task (Lin \& Murray, 2013, 2015a). The study was approved by the local Institutional Review Board.

Apparatus The stimuli were presented on a 19-in CRT monitor (ViewSonic G90fB; refresh rate $=60 \mathrm{~Hz}$; resolution $=1$, $024 \times 768$ pixels). Participants sat approximately $50 \mathrm{~cm}$ from the monitor with their heads positioned in a chin rest in an almost dark room (no lighting except from the computer and the monitor).

Procedure and design Participants were first trained to fixate at a central point using a flicker procedure, which has been shown to improve their subsequent ability to maintain central eye fixation (Guzman-Martinez, Leung, Franconeri, Grabowecky, \& Suzuki, 2009). During this 2-min flicker training session, a stationary dot appeared in the middle of a flicking square image; the image consisted of black and white pixels that flickered in counterphase (that is, each pixel alternated between black and white across frames). Participants were asked to fixate at the dot, and real-time feedback on fixation stability was embodied by the appearance of the background image - a flash of visual noise on the image would appear whenever the fixation deviated from the central dot.

After the fixation training, participants proceeded to the main experiment: the dot-detection session. Figure 1a illustrates the procedure. Each trial started with a fixation point - a combination of a bull's-eye and crosshairs (diameter of inner annulus $=0.23^{\circ}$; diameter of outer annulus $=0.69^{\circ}$; luminance $=49.2 \mathrm{~cd} / \mathrm{m}^{2}$ for crosshairs, $0.14 \mathrm{~cd} / \mathrm{m}^{2}$ for bull'seye)-appearing for $1,000 \mathrm{~ms}$ in the center of a gray background (luminance $=12.0 \mathrm{~cd} / \mathrm{m}^{2}$ ). The fixation was followed by a blank screen for $200 \mathrm{~ms}$, which was then followed by the cue-target display.

The cue in the cue-target display lasted for $16.7 \mathrm{~ms}$. It manifested as 1) one single abrupt-onset annulus (diameter $=$ $3^{\circ}$, width $=0.25^{\circ}$ ) in red (luminance $=10.8 \mathrm{~cd} / \mathrm{m}^{2} ; x=0.600, y$ $\left.=0.326, u^{\prime}=0.420, v^{\prime}=0.514\right), 6^{\circ}$ away from the fixation to the left or to the right; 2) two of the above annuli, one each on the left and right; or 3) no annulus, just a blank screen. After the cue disappeared, a blank screen - either 0 ms (i.e., absent; as in Fig. 1a), $300 \mathrm{~ms}$, or $600 \mathrm{~ms}$ - was inserted to create a CTOA of $16.7 \mathrm{~ms}, 316.7 \mathrm{~ms}$, or $616.7 \mathrm{~ms}$. This was immediately followed by three black annuli (luminance $=0.14 \mathrm{~cd} /$ $\mathrm{m}^{2}$ ) on the left, center, and right of the fixation for $283.3 \mathrm{~ms}$. On $80 \%$ of the trials, a black target dot (diameter $=1.9^{\circ}$; luminance $=0.14 \mathrm{~cd} / \mathrm{m}^{2}$ ) also appeared, randomly within the left or right annulus and at the same time as the annuli but lasting only for the first $83.3 \mathrm{~ms}$ (for the remaining $200 \mathrm{~ms}$, the dot disappeared and only the three annuli remained onscreen). On $20 \%$ of the trials, the dot did not appear. The task was to detect the presence of the dot by pressing a button as quickly as possible when it appeared, but refrain from response when it was absent. The trial ended when a response was made or $1 \mathrm{~s}$ after the offset of the annuli, whichever was earlier. Feedback was provided: an incorrect response was followed by two

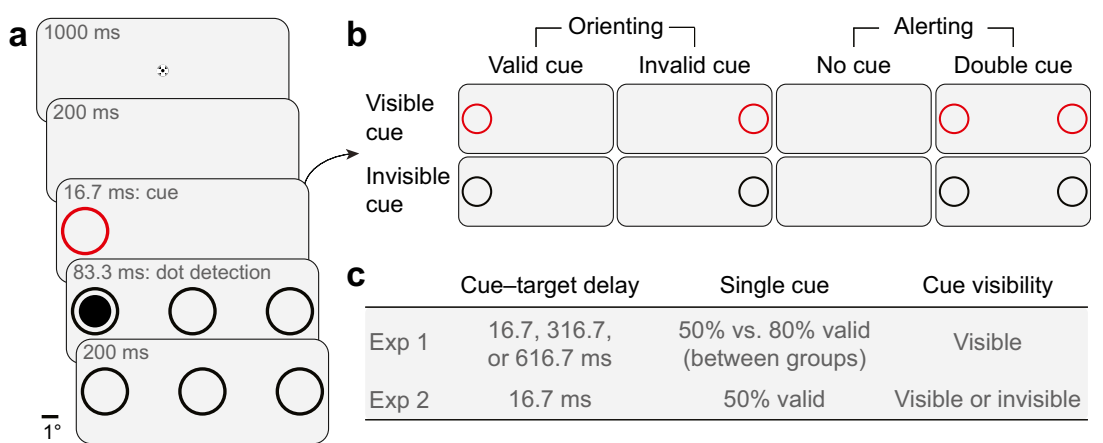

Fig. 1 Experimental design. (a) Task. For $80 \%$ of the trials, a target dot briefly appeared, randomly within the left or right annulus. For $20 \%$ of the trials, the dot was not presented. The task was to press a button as quickly as possible when the dot appeared - but refrain from response if it did not appear. (b) Manipulations of cue visibility and cue location. The annular cue could be in red, released from masking by the subsequent black annuli (hence, visible), or in black, masked by the annuli (hence, invisible). For both visible and invisible cues, there were four types of cues within each block: valid cue (the target appeared at the cued location); invalid cue (the target appeared at the uncued location); no cue (blank screen); double cue (the cue appeared on both the left and right). The orienting effect was calculated by subtracting the reaction time (RT) in the valid cue condition from the invalid cue condition; the alerting effect, the double cue condition from the no cue condition. (c)
Summary of key factors manipulated in Experiments 1 and 2. Experiment 1 used only visible cues but varied two factors: 1 ) the cue to target onset delay (within participants and within blocks) - either $16.7 \mathrm{~ms}$ (as depicted in panel a), $316.7 \mathrm{~ms}$, or $616.7 \mathrm{~ms}$ - as determined by the duration of the blank screen inserted in between the cue and target displays; and 2) the task-relevance of the single annular cue (between participants), such that the location of the cue annulus was either uninformative regarding the location of the target dot (50\% valid; the uninformative-cue group) or informative of the target location ( $80 \%$ valid; the informative-cue group). Experiment 2 used only the $16.7 \mathrm{~ms}$ cue-target delay and included uninformative single cues, but varied the cue visibility (within participants but across blocks). Cue visibility was measured at the end of the experiment in a separate session, by asking participants to directly discriminate the cue location (see text) 
tones (each lasting $200 \mathrm{~ms}$ with a 5-ms interval in between) and a 5-s timeout (a blank screen).

Participants practiced 24 trials before completing 10 blocks of experimental trials, 90 trials each, for a total of 900 trials. Each block of 90 trials consisted of 18 target-absent trials $(20 \%)$ and 72 target-present trials $(80 \%)$. There were four types of target-present trials, as determined by the annular cue and the target within it (Fig. 1b): 1) a valid-cue trial, in which the target dot appeared within the cued annulus; 2) an invalidcue trial, in which the dot appeared within the uncued annulus; 3) a no-cue trial, in which none of the annuli was cued; or 4) a double-cue trial, in which both the left and right annuli were cued. Each block of 72 target-present trials consisted of 6 nocue trials, 6 double-cue trials, and either 30 valid-cue trials and 30 invalid-cue trials (for the uninformative-cue group) or 48 valid-cue trials and 12 invalid-cue trials (for the informativecue group). In other words, in eliciting the orienting effect, the location of the red cue was uninformative with respect to the target location for the uninformative-cue group (50\% valid), but it was informative for the informative-cue group (80\% valid; Fig. 1c). For both groups, the three CTOAs $-16.7 \mathrm{~ms}$, $316.7 \mathrm{~ms}$, and $616.7 \mathrm{~ms}$ - were randomized within each block.

In total, for the uninformative-cue group, there were 20 nocue trials, 20 double-cue trials, 100 valid-cue trials, and 100 invalid-cue trials for each CTOA; for the informative-cue group, there were 20 no-cue trials, 20 double-cue trials, 160 valid-cue trials, and 40 invalid-cue trials for each CTOA.

Data analysis The analysis focused on the reaction times (RTs) in correctly detecting the target (i.e., when the button was correctly pressed in response to a target; also called hit trials). False-alarm trials (when no target was presented but the button was pressed) and trials with anticipatory responses (RT < $100 \mathrm{~ms}$, as in Lin \& Murray, 2014) were excluded from the RT analysis (a cutoff of RT $\leq 250 \mathrm{~ms}$ yielded the same patterns of results, as detailed in the Supplemental Online Materials §1).

With respect to accuracy, based on prior work (Lin \& Murray, 2015a), we expected the appearance of visible cues (in both single-cue and double-cue trials) to induce more false alarms than no-cue trials; this difference in false alarms will be referred to as cue-induced false alarms. For orienting effects, because invalid-cue trials and valid-cued trials were both single-cue trials, their subtraction was unaffected by cueinduced false alarms - they shared the same term in falsealarm rate. Conversely, for alerting effect, cue-induced false alarms - i.e., the difference between double-cue trials and nocue trials in false alarms - were expected, and the question to be addressed was how CTOA and cue task-relevance modulated cue-induced false alarms. The raw data and the corresponding Matlab analysis codes can be accessed online at: https://osf.io/hpynm.

\section{Results}

Effects of task relevance and CTOA on orienting and alerting Figure 2 shows the main results. For the orienting effect (Fig. 2, top), a $3 \times 2$ repeated-measures ANOVA with the factors CTOA (16.7 ms, $316.7 \mathrm{~ms}$, vs. $616.7 \mathrm{~ms}$ ) and group (uninformative-cue group vs. informative-cue group) revealed only a significant main effect of CTOA, $F(2,76)=$ $36.33, p<0.001, \eta_{p}^{2}=0.49$, without a main effect of group, $F(1,38)=2.23, p=0.143, \eta_{p}^{2}=0.06$, or their interaction $F(2$, 76) $=0.51, p=0.601, \eta_{p}^{2}=0.01$. Further analysis on the CTOA main effect showed that the orienting effect was modulated by the CTOA: at $16.7 \mathrm{~ms}$, there was a facilitation effect at the cued location relative to the uncued location, $8.9 \mathrm{~ms}$, $t(39)=3.89, p<0.001$, Cohen's $d=0.62$; but at $316.7 \mathrm{~ms}$ and $616.7 \mathrm{~ms}$, the effect was reversed, reflecting inhibition of return (Klein, 2000), $-12.8 \mathrm{~ms}, t(39)=-5.45, p<.001, d=$ $-0.86 ;-13.9 \mathrm{~ms}, t(39)=-6.25, p<0.001, d=-0.99$.

Turning to the alerting effect from the double cue (Fig. 2, bottom), a repeated-measures ANOVA with the factors CTOA and group revealed not only a significant main effect of CTOA, $F(2,76)=4.86, p=0.010, \eta_{p}^{2}=0.11$, but also a significant main effect of group, $F(1,38)=4.21, p=0.047$, $\eta_{p}^{2}=0.10$, without their interaction, $F(2,76)=0.78, p=0.464$, $\eta_{p}^{2}=0.02$. The CTOA main effect reflected that the alerting effect, like the orienting effect, also was modulated by the CTOA, but in a different way. Rather than reversing its direction, the alerting effect was in the same direction across the 3 CTOAs: at $16.7 \mathrm{~ms}$, the alerting effect was weak, $7.8 \mathrm{~ms}, t(39)$

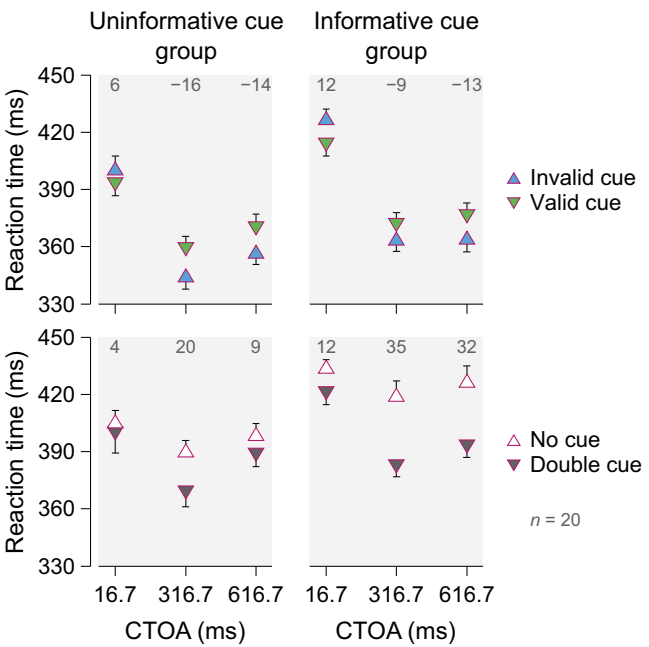

Fig. 2 Effects of cue-target onset asynchrony (CTOA) and cue taskrelevance on attentional orienting and alerting. In both uninformativecue and informative-cue groups, valid cues speeded up responses when the CTOA was $16.7 \mathrm{~ms}$ but slowed down responses when CTOAs were $316.7 \mathrm{~ms}$ and $616.7 \mathrm{~ms}$ (top). The double cue speeded up responses compared with when there was no cue, but importantly this alerting effect was more pronounced in blocks mixing informative (relative to uninformative) single cues and when the CTOA was lengthened (bottom). Orienting and alerting effects (in $\mathrm{ms}$ ) are shown on the top. Error bars (one-sided) are standard errors of the mean 
$=1.52, p=0.136, d=0.24$; at $316.7 \mathrm{~ms}$ and $616.7 \mathrm{~ms}$, the alerting effect increased in magnitude, $27.4 \mathrm{~ms}, t(39)=4.64$, $p<0.001, d=0.73 ; 20.4 \mathrm{~ms}, t(39)=4.05, p<0.001, d=0.64$. The group main effect revealed a greater alerting effect in the informative-cue group $(26.3 \mathrm{~ms})$ than in the uninformativecue group (10.8 ms), a novel observation not previously reported. Further analysis as detailed in Appendix 1 revealed that these RT results could not be explained by speed-accuracy tradeoffs.

Sensory summation Given that the no-cue, single-cue, and double-cue trials differed in the cue, a concern is whether performance differences between conditions might reflect different sensory summations between cue and target processing. The design here minimized sensory summation, as the cue and the target had no spatial overlap (Fig. 1a). This is further corroborated by the results in orienting and alerting effects. First, in the orienting effect, sensory summation would not predict inhibition at the longer CTOAs, which instead reflects a classic pattern of stimulus-driven orienting from abruptonset cues (Posner \& Cohen, 1984). Second, in the alerting effect, sensory summation would predict the strongest alerting effect to appear at the shortest CTOA because of sensory dissipation over time, but this prediction is opposite to the actual observation (Fig. 2). Third, also in contrast to the sensory summation account, on average RT was faster rather than slower when the CTOA was lengthened (Fig. 2; see the Supplemental Online Materials $\S 2$ for the ANOVA results). These results therefore indicate that the ANT tapped into attentional processes, in keeping with a large body of prior work using this method.

Cue-induced false alarms Consistent with prior work (Lin \& Murray, 2015a), there were more false alarms in double-cue trials than in no-cue trials, implicating cue-induced false alarms (Table 1). A repeated-measures ANOVA with the factors CTOA and group on cue-induced false alarms - i.e., on the difference in false alarms between double-cue trial and nocue trials - revealed a significant main effect of CTOA, $F(2$, 76) $=6.52, p=0.002, \eta_{p}^{2}=0.15$, without a main effect of group, $F(1,38)=0.08, p=0.782, \eta_{p}^{2}<0.01$, or their interaction, $F(2,76)=0.08, p=0.928, \eta_{p}^{2}<0.01$. The CTOA main effect showed that cue-induced false alarms were strongly reduced when the CTOA was lengthened (16.7 ms: $20.5 \%$; $316.7 \mathrm{~ms}: 8.6 \%$; $616.7 \mathrm{~ms}: 3.5 \%$ ). A similar pattern was found when comparing single-cue trials and no-cue trials, showing a significant main effect of CTOA, $F(2,76)=6.22$, $p=0.003, \eta_{p}^{2}=0.14(16.7 \mathrm{~ms}: 18.3 \%$; $316.7 \mathrm{~ms}: 9.4 \%$; $616.7 \mathrm{~ms}: 7.3 \%$ ). (For the ANOVA results on the absolute false-alarm rates as opposed to cue-induced false alarms, see the Supplemental Online Materials §2.)

\section{Discussion}

Two main findings emerge from Experiment 1. First, whereas increasing CTOA reversed the direction of the orienting effect from facilitation to inhibition, increasing CTOA strengthened the alerting effect and reduced cue-induced false alarms. Second, whereas the informativeness of the single cue did not modulate bottom-up orienting effects, it strongly affected alerting: the alerting effect was enhanced when the double cue was mixed with informative - relative to uninformative - single cues.

Additional analysis indicated that these results could not be explained by sensory summation or speed-accuracy tradeoffs. Eye movements also are unlikely to account for the results. First, given that the double cue appeared at both sides of the fixation and provided no spatial information regarding the target, there was little incentive for eye movements, which in any case would not help performance. Second, the informativeness of the single cue did not alter the orienting effects, both in terms of facilitation at the shortest CTOA - which was too short for executing a saccade — and inhibition at the longer CTOAs, suggesting that systematic eye movements were not likely.

What might explain the limited influence of cue informativeness on the orienting effects, both at the shortest CTOA and at the longer CTOAs? The 16.7-ms CTOA may be too short for endogenous orienting to manifest, because at this timescale the orienting effect is likely to be dominated by rapid and reflexive exogenous attention from the abruptonset cue (Cheal \& Lyon, 1991). At longer CTOAs of $316.7 \mathrm{~ms}$ and $616.7 \mathrm{~ms}$, the inhibition of return effect observed there indicated bottom-up attentional capture by the abrupt-onset cue in both the informative-cue and uninformative-cue groups. This is evidence against the proposal that inhibition of return is exclusive to uninformative cues (Wright \& Richard, 2000), and suggests that inhibition may mask the effect from endogenous orienting. ${ }^{2}$ That is, although task relevance and bottom-up capture jointly determine attention behavior from abrupt-onset cues, in the case of orienting, inhibition of return at longer CTOAs - arising from exogenous orienting - may mask the effect of task relevance.

In this context, how could we know that the manipulation of cue informativeness was successful in modulating voluntary or endogenous attention? The strong modulation effect of cue informativeness on the alerting effect, particularly at the longer CTOAs of $316.7 \mathrm{~ms}$ and $616.7 \mathrm{~ms}$, provides some indirect evidence.

\footnotetext{
${ }^{2}$ Although not used in the present study, intermediate CTOAs between $16.7 \mathrm{~ms}$ and $316.7 \mathrm{~ms}$ may offer a better chance to detect a modulation effect on orienting by cue informativeness. However, we suspect that the modulation effect is likely to be modest, considering the strong, dominant effect of bottom-up attentional capture and considering the still relatively short CTOAs for effective top-down guidance.
} 
Table 1 Miss (M), false alarm (FA), sensitivity $\left(d^{\prime}\right)$, and criterion $(c)$ in Experiments 1 and 2

\begin{tabular}{|c|c|c|c|c|c|c|c|c|c|c|c|c|}
\hline \multirow[b]{2}{*}{ Experiment and condition } & \multicolumn{4}{|l|}{ No cue } & \multicolumn{4}{|c|}{ Single cue (valid|invalid) } & \multicolumn{4}{|c|}{ Double cue } \\
\hline & $\mathrm{M}(\%)$ & FA $(\%)$ & $d^{\prime}$ & $c$ & $\mathrm{M}(\%)$ & FA $(\%)$ & $d^{\prime}$ & $c$ & $\mathrm{M}(\%)$ & FA $(\%)$ & $d^{\prime}$ & $c$ \\
\hline \multicolumn{13}{|c|}{ Exp 1: Uninformative-cue group } \\
\hline $16.7 \mathrm{~ms}$ & 0.3 & 6.1 & 5.4 & -0.3 & $1.1 \mid 1.4$ & 20.3 & $3.6 \mid 3.5$ & $-0.8 \mid-0.8$ & 1.1 & 27.0 & 4.0 & -0.8 \\
\hline $316.7 \mathrm{~ms}$ & 0.6 & 8.1 & 5.2 & -0.3 & $1.2 \mid 0.8$ & 16.7 & $3.7 \mid 3.9$ & $-0.8 \mid-0.9$ & 0.6 & 16.3 & 4.6 & -0.6 \\
\hline $616.7 \mathrm{~ms}$ & 1.1 & 5.1 & 5.4 & -0.2 & $1.6 \mid 1.7$ & 12.7 & $3.8 \mid 3.8$ & $-0.5 \mid-0.6$ & 3.4 & 10.1 & 4.7 & -0.1 \\
\hline \multicolumn{13}{|c|}{ Exp 1: Informative-cue group } \\
\hline $16.7 \mathrm{~ms}$ & 1.6 & 6.1 & 5.3 & -0.1 & $1.7 \mid 2.5$ & 28.5 & $3.1 \mid 3.2$ & $-0.9 \mid-0.9$ & 3.6 & 26.0 & 3.4 & -0.7 \\
\hline $316.7 \mathrm{~ms}$ & 1.3 & 7.1 & 5.2 & -0.2 & $1.2 \mid 1.6$ & 17.3 & $3.6 \mid 3.8$ & $-0.7 \mid-0.7$ & 0.4 & 16.0 & 4.7 & -0.7 \\
\hline $616.7 \mathrm{~ms}$ & 1.3 & 7.1 & 5.3 & -0.2 & $2.4 \mid 2.9$ & 14.0 & $3.4 \mid 3.5$ & $-0.5 \mid-0.6$ & 4.4 & 9.1 & 4.9 & -0.0 \\
\hline \multicolumn{13}{|l|}{ Exp 2} \\
\hline Visible cue & 0.5 & 9.8 & 4.7 & -0.5 & $0.4 \mid 0.6$ & 27.6 & $3.6 \mid 3.4$ & $-1.1 \mid-1.1$ & 1.2 & 26.4 & 3.3 & -1.0 \\
\hline Invisible cue & 1.1 & 11.8 & 4.4 & -0.6 & $1.5 \mid 0.6$ & 15.3 & $4.0 \mid 4.2$ & $-0.7 \mid-0.8$ & 1.3 & 15.9 & 4.2 & -0.7 \\
\hline
\end{tabular}

Note: $d^{\prime}$ and $c$ were calculated as follows. $d^{\prime}=z(1-$ miss $)-z$ (false alarm). $c=-(z(1-$ miss $)+z($ false alarm $)) / 2$

In principle, the orienting effect could reflect either a modulation in perceptual processing or a modulation in decision weighting, such as a higher weight to the cued location than to the uncued location (Eckstein, Shimozaki, \& Abbey, 2002). The decision-based account, however, is not completely compatible with our results. First, this account would not predict the reversing of orienting from facilitation to inhibition when the CTOA was increased. Second, it would predict the orienting effect to be dependent on cue informativeness, which as noted above was not the case here. Therefore, the orienting effect observed here likely represents a perceptual effect rather than a decision effect (Z. L. Lu \& Dosher, 1998).

A previous study found no alerting effect when no-cue trials and double-cue trials were mixed with $100 \%$ valid single-cue trials, but there was a significant alerting effect when mixed with $75 \%$ valid single-cue trials (Greene et al., 2008). However, because no statistics were reported, it was not clear whether the difference between the two conditions was statistically significant. More importantly, the study was confounded by several other differences between the two informativeness conditions, including different stimuli types and target durations. Indeed, given that the single cue was highly informative in both cases (100\% and $75 \%$ valid), the study did not appear to be set up to test the role of context-based task-relevance on alerting.

\section{Experiment 2}

Experiment 1 shows that alerting can be enhanced by increasing the CTOA and by the mixture of informative single cues-reflecting a global, contextual influence. Consequently, within a short temporal window following its appearance, a salient but task-irrelevant cue is unable to reliably alert attention. Being salient, in other words, is not sufficient for a cue to alert attention.

Two distinct accounts may explain the limiting condition for the alerting effect at the 16.7-ms CTOA. If we assume that alerting must rely solely on endogenous strategies, then $16.7 \mathrm{~ms}$ is simply too short for an alerting effect to develop. This would predict the absence of an alerting effect as long as the CTOA is kept at $16.7 \mathrm{~ms}$ - the fundamental constraint is a temporal one. On the other hand, if we presume a stimulusdriven component in alerting, then the culprit may lie in some negative effect accompanying the brief CTOA that counteracts and masks the stimulus-driven alerting effect. If so, an alerting effect may manifest itself at the 16.7-ms CTOA when the counteracting force is reined.

The latter possibility - that the 16.7-ms CTOA may induce some counteracting force against cueing-receives some support from a recent study of exogenous orienting (Lin \& Murray, 2015a). There it was found that when the cue and the target are spatially and temporally close to each other, the task of extracting the task-relevant information (the target) may require or benefit from suppressing the salient, taskirrelevant information (the cue). The inhibition of the cue was found to depend crucially on the cue being visiblehence the term "awareness-dependent inhibition." We reasoned that if the same mechanism is at play here, then by removing awareness we might be able to minimize inhibition and reveal an alerting effect. The question therefore becomes, what happens when the uninformative salient cue is rendered subliminal? When a salient, visible cue fails to elicit an alerting effect, can we nevertheless observe an alerting effect with a nonsalient, subliminal cue?

This question is addressed in Experiment 2 by using the shortest CTOA $(16.7 \mathrm{~ms})$ and by mixing the double cue with an uninformative single cue-i.e., the same limiting condition 
for conscious alerting as identified in Experiment 1. Crucially, we also varied the cue visibility: the cue was visible (in red, as before) in half of the blocks but invisible (in black, hence masked by the trailing black annuli) in the other half of the blocks (Fig. 1c). To gauge participants' awareness of the cue, we further included objective forced-choice tests after the main experiment, which measured participants' accuracy in choosing the location (left vs. right) and interval $\left(1^{\text {st }}\right.$ vs. $\left.2^{\text {nd }}\right)$ of the cue.

\section{Method}

Participants A new group of 20 participants with normal or corrected-to-normal vision were recruited (11 females, 9 males; mean age $=19.8$ years).

Procedure and design Experiment 2 was similar to Experiment 1 except for two major changes. The first major change was on the experimental manipulation (Fig. 1c). Only the shortest CTOA $(16.7 \mathrm{~ms})$ was used. Cues were uninformative, with equal proportions of valid-cue trials, invalid-cue trials, no-cue trials, and double-cue trials. Most importantly, the cue could be either visible (in red color, released from masking) or invisible (black, strongly masked) in different blocks (five consecutive blocks each, with the order counterbalanced between participants). Participants practiced 12 trials before completing 800 experimental trials (10 blocks $\times 80$ trials). In total, there were eight conditions - no-cue trials, double-cue trials, valid-cue trials, and invalid-cue trials in visible and invisible conditions - each including 80 trials, for a total of 640 trials. The remaining 160 trials were targetabsent trials.

With the addition of the invisible cues, the second major change was on the experimental procedure. That is, to make sure that the invisible cues were indeed invisible, at the end of the experimental session we asked participants to discriminate directly the cue location in two separate tests. The first test focused on whether participants were aware of the single cue (i.e., the valid-cue and invalid-cue trials). In 80 trials, the same subliminal cue as in the main experiment was presented either on the left or on the right, and participants were asked to indicate the cue's location as accurately as possible with no constraint on response time. Subsequently, a dot-previously designated as the target but now irrelevant for the task-was equally likely to appear within the left or right annulus. Participants practiced 16 trials on the same task but using visible cues to make sure that they understand and properly perform the task-important because only then did chance performance in the formal session allow for meaningful interpretation (Lin \& Murray, 2014). The second test focused on whether participants were aware of the double cue. A double-cue trial and a no-cue trial were presented sequentially as a pair of trials, with the double-cue trial presented first in half of the pairs; participants were asked to indicate which interval — first or second trial — contained the double cue. An irrelevant dot was equally likely to appear within the doublecue trial or no-cue trial in each trial-pair. There were 80 trialpairs in total. As before, participants practiced 16 trial-pairs on the same task but with visible cues. The trial structure was the same as that in the dot-detection session.

\section{Results}

Alerting from invisible - but not visible — cues The criterion for "aware" versus "unaware" was based on the binomial test conducted on the accuracy results in the awareness tests: $p>0.05$ (one-tailed), unaware; $p \leq 0.05$, aware. Only when performance was classified as aware in the practice session (visible-cue targets) but unaware in the formal session (invisible-cue targets) across both the location and interval tests were participants included in further data analysis. Eighteen participants were thus included. Their group performance in the formal session also did not differ from chance (left-right location test: $M=$ $50.4 \%$ correct, $S D=3.7 \%, t(17)=0.47, p=0.64$, twotailed; first-second interval test: $M=51.2 \%$ correct, $S D=$ $3.6 \%, t(17)=1.49, p=0.15)$, which further suggests that they were unable to discern the cue.

Prior work has shown that cues that are rendered invisible can still influence attention orienting (for reviews, see Lin \& He, 2009; Mulckhuyse \& Theeuwes, 2010; van Boxtel, Tsuchiya, \& Koch, 2010). In particular, visible and invisible exogenous cues can give rise to orienting effects that are of similar magnitude (Lin \& Murray, 2015a). This finding was replicated here. A repeated-measures ANOVA with the factors validity (valid vs. invalid) and visibility (visible vs. invisible) revealed significant main effects of validity, $F(1,17)=56.25$, $p<0.001, \eta_{p}^{2}=0.77$, and visibility, $F(1,17)=4.76, p=0.044$, $\eta_{p}^{2}=0.22$, without their interaction, $F(1,17)=0.39, p=0.395$, $\eta_{p}^{2}=0.04$. The validity main effect reflected a facilitation orienting effect at the cued location relative to the uncued location, both for the visible cue, $12.4 \mathrm{~ms}, t(17)=5.25, p<$ $0.001, d=1.24$, and for the invisible cue, $15.6 \mathrm{~ms}, t(17)=$ $5.39, p<.001, d=1.27$ (Fig. 3).

The critical question concerns the alerting effect. A repeated-measures ANOVA with the factors cue (double-cue vs. no-cue) and visibility (visible vs. invisible) revealed significant main effects of cue, $F(1,17)=7.84, p=0.012, \eta_{p}^{2}=$ 0.32 , visibility, $F(1,17)=11.75, p=0.003, \eta_{p}^{2}=0.41$, and their interaction, $F(1,17)=7.73, p=0.013, \eta_{p}^{2}=0.31$. The interaction effect reflected that the visible cue did not evoke an alerting effect as in Experiment 1, $2.1 \mathrm{~ms}, t(17)=0.55, p=$ $0.586, d=0.13$; in contrast, with the same CTOA, the invisible cue evoked a robust alerting effect, $12.8 \mathrm{~ms}, t(17)=4.70$, $p<0.001, d=1.11$. As detailed in Appendix 2, these RT effects did not reflect speed-accuracy tradeoffs. These results 


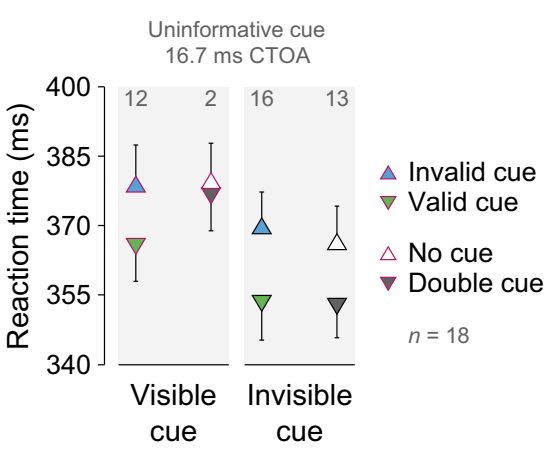

Fig. 3 Invisible cues alert attention where visible cues fail. Under the same condition - when the single cue was uninformative, at a cue-target onset asynchrony (CTOA) of $16.7 \mathrm{~ms}$ - the orienting effect was similar regardless of cue visibility, but the alerting effect was present only in the invisible cue, absent in the visible cue. Orienting and alerting effects (in $\mathrm{ms}$ ) are shown on the top. Error bars (one-sided) are standard errors of the mean

provide a novel demonstration that a nonsalient, subliminal cue can elicit an alerting effect where a salient cue fails to do so.

\section{Reduction of cue-induced false alarms from invisible cues} Consistent with prior work (Lin \& Murray, 2015a), in doublecue trials, the cue-induced false-alarm rate was much higher when the cue was visible $(16.5 \%$; Table 1$)$ than invisible $(4.0 \%), t(17)=3.85, p=0.001, d=0.91$. A similar pattern was found comparing single-cue trials and no-cue trials (visible cue: $17.8 \%$; invisible cue: $3.5 \%$; $t(17)=4.00, p<0.001$, $d=0.94)$.

Therefore, rendering the cue invisible not only rescued the alerting effect but also strongly reduced cue-induced false alarms. These two effects were dissociable, as there was no correlation between the increase in the alerting effect and the reduction in the cue-induced false alarms across participants, Pearson's $r=-0.34, p=0.162$. Nor did the magnitude of the unconscious alerting effect correlate with the amount of cueinduced false alarms, $r=-0.07, p=0.787$.

\section{Dissociating unconscious alerting from unconscious} orienting and conscious alerting The demonstration of an unconscious but not a conscious alerting effect raises the question of how visual awareness may modulate attention. According to some models of attention, orienting and alerting (as well as executive control) are independent components of conscious attention (Petersen \& Posner, 2012), but whether this relation is a particular case confined only to conscious attention or it extends to unconscious attention remains largely unexplored.

Our experiment was not designed to specifically address this issue, because the statistical power was weak for detecting small correlations. Given a one-tailed $\alpha$ level of 0.05 , power of .08 , detecting a correlation of $r=0.56$ requires a sample size $n$ of 18 , but detecting a weaker correlation would require a much larger sample size. For example, detecting $r=0.4$ requires $n$ of $37 ; r=0.3, n$ of $67 ; r=0.2, n$ of 153 . Nevertheless, our data could provide some preliminary results regarding this important issue. In particular, because participants were tested with both visible and invisible cues, the experiment allowed us to evaluate the correlation of attention components both within and across visibility conditions.

Consistent with previous research in conscious attention (Fan et al., 2002; Fernandez-Duque \& Posner, 1997), we found that conscious orienting effects did not correlate with conscious alerting effects, $r=-0.26, p=0.293$ (Fig. 4, upper left). But more importantly, we found no significant correlation between unconscious orienting effects and unconscious alerting effects, $r=0.12, p=0.627$ (Fig. 4, upper right), suggesting that orienting and alerting may be dissociable components not only in conscious attention but also in unconscious attention.

Going beyond correlations within visibility conditions, next we asked, what is the relation between conscious and unconscious attention? The fact that an unconscious alerting effect can manifest itself without a corresponding conscious alerting effect suggests that conscious and unconscious attention must involve nonoverlapping neural mechanisms, at least in the case of alerting. Consistent with this possibility, we found that unconscious orienting effects did not correlate with conscious orienting effects, $r=-0.003, p=0.990$ (Fig. 4, lower left). The same also held for unconscious and conscious alerting effects, $r=0.33, p=0.184$ (Fig. 4, lower right). These results from alerting and orienting converge to suggest that
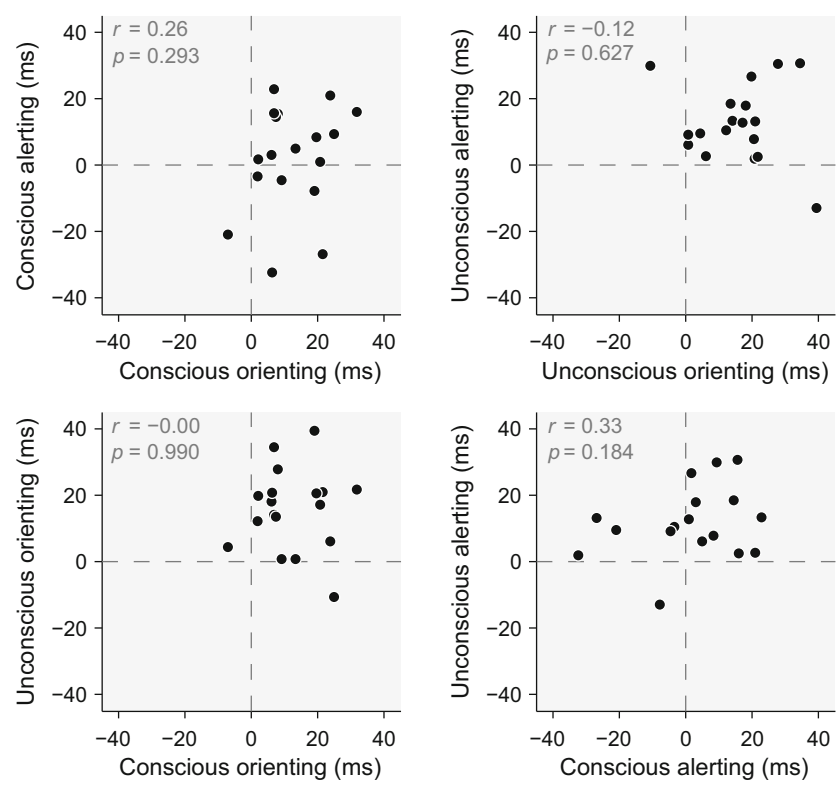

Fig. 4 Lack of correlations between orienting and alerting effects and between conscious and unconscious effects. Between components of attention, orienting did not correlate with alerting for both conscious (upper left) and unconscious attention (upper right). Between levels of visibility, the conscious effect did not correlate with the unconscious effect for both orienting (lower left) and alerting (lower right) 
conscious attention and unconscious attention may emerge from nonidentical mechanisms.

Direct evidence for awareness-dependent inhibition Why was there an unconscious alerting effect without a corresponding conscious alerting effect? This dissociation can be understood if we assume that conscious processing comes with an exclusive cost that does not apply to unconscious processing. One possibility is that when a salient cue is spatially and temporally close to the target but is uninformative for the task, wariness of potential distraction from the cue triggers the executive-control system to inhibit the cue (Lin \& Murray, 2015a; Olivers \& Meeter, 2008). This awareness-dependent inhibition account finds some support from motion perception, where task-irrelevant background random dots were found to be under inhibitory control only when they were suprathreshold (Tsushima, Sasaki, \& Watanabe, 2006). More direct support comes from a recent finding in attentional orienting, where repeatedly cueing the same location was found to induce a persistent attentional bias against this location even when the cue was no longer present, but this pattern manifested itself only when the cue was visible (Lin \& Murray, 2015a).

To further evaluate the awareness-dependent inhibition account, we compared performance between visible-cue blocks and invisible-cue blocks. We reasoned that if wariness of distraction induces a propensity to inhibit the source of distraction, then this propensity for inhibition should be stronger in visible-cue blocks than in invisible-cue blocks, resulting in slower mean RT in visible- than invisible-cue blocks. The results confirm this conjecture (Fig. 3, top): performance was slower by $14.6 \mathrm{~ms}$ in visible-cue blocks than in invisible-cue blocks, $t(17)=2.88, p=0.010, d=0.68$.

Perhaps more telling were trials in which the cues were absent, because these no-cue trials were identical between the visible- and invisible-cue blocks. In other words, based on the awareness-dependent inhibition account, one may expect RT to be slower for the no-cue trials within the visiblethan invisible-cue blocks. Consistent with this expectation, we found that the otherwise identical no-cue trials were slower by $13.2 \mathrm{~ms}$ in visible-cue blocks than in invisible-cue blocks, $t(17)=2.40, p=.028, d=0.57$ (Fig. 3, top). These results provide new evidence for the awareness-dependent inhibition account, revealing a generalized, global side effect of cue awareness on RT.

Thus, when trying to inhibit the cue but only with partial success, participants under the influence of awarenessdependent inhibition exhibit two behavioral footprints: an increase in false alarm rates, arising from a difficulty in fully inhibiting the cue (i.e., cue-induced false alarms, as documented above); and a corresponding increase in RT, arising from the mobilization of inhibitory processes. This pattern is consistent with the effect of CTOA in Experiment 1: increasing the CTOA - particularly from $16.7 \mathrm{~ms}$ to $316.7 \mathrm{~ms}$ - not only reduces false-alarm rates (Table 1) but also reduces RT (Fig. 2; see Supplemental Online Materials $§ 2$ for statistical results).

\section{Discussion}

Using uninformative cues and a CTOA of $16.7 \mathrm{~ms}$, Experiment 2 made three observations. First, visible and invisible exogenous cues elicited similar orienting effects, but they differed markedly in the alerting effect: whereas visible cues failed to elicit alerting, masking the cue from awareness restored the alerting effect (and reduced the cue-induced false alarm rates). Second, orienting and alerting effects appeared to be not significantly correlated, both for visible and invisible cues. Effects of conscious and unconscious attention also were not correlated, suggesting nonidentical mechanisms. Third, the overall RT - as well as the RT for the identical, no-cue trials - was slower in visible-cue blocks than in invisible-cue blocks, indicating awareness-dependent inhibition.

These results help constrain the interpretation of the orienting effect - whether it may simply reflect a lower response criterion in the cued location than in the uncued location. On the surface this account is difficult to refute because in the detection task, it was not possible to separate false alarm trials for valid and invalid trials: when a button was incorrectly pressed on a target-absent trial, it was not clear whether the false alarm was toward the cued or uncued location. For this reason, the criterion analyses on orienting effects (as detailed in Appendix 2) were based on a shared false-alarm term. However, the fact that the orienting effect manifested itself when the cue was rendered invisible provides strong evidence against this criterion-based account. If participants lowered their criteria for responding to the expected (cued) location than to the unexpected (uncued) location, such a strategy not only was not helpful for the task - the cue was uninformative - but was not possible in the first place when the cue was invisible.

\section{General discussion}

Salient stimuli in the environment- those that stand out conspicuously - are generally believed to heighten our momentary response readiness to the environment. We show that being salient is not sufficient for a task-irrelevant cue to transiently alert attention, nor is it necessary. Alerting was strengthened when the CTOA was increased and when the double cue was mixed with an informative single cue (and thereby rendered task-relevant based on the context). As a result, within a short temporal window after its appearance, a salient but task-irrelevant cue was unable to reliably alert attention. Importantly, under this limiting condition, the alerting effect manifested itself when the cue was rendered 
subliminal. The counterintuitive cost of awareness reflects awareness-dependent inhibition (Lin \& Murray, 2015a), arising from the inhibition of salient, task-irrelevant cues that are spatiotemporally close to the target.

Together with the recent demonstration of cue-induced inhibition in orienting (Lin \& Murray, 2015a), these findings lead us to propose that a simple visual cue can produce a much more complex attentional effect than traditionally thought, as determined by how the cue differentially engages three components of attention - orienting, alerting, and inhibition (Table 2). Below, we first describe the three-component perspective of exogenous cueing. Then, under this general perspective, we present the view that alerting can be defined and studied as general arousal and specific response readiness, and it is controlled by both internal, top-down expectation and external, bottom-up stimulus properties. We go on to illustrate how alerting is tied to 1) temporal orienting, 2) attentional capture, and 3) spatial orienting. Finally, we end with a discussion on the measurement of attention in applied and clinical settings and a general conclusion.

Three components in exogenous cueing The appearance of an exogenous cue - be it a single cue or a double cue - captures spatial attention to the cued region. We propose that this seemingly simple act of capture can give rise to three attentional effects: 1) a spatial orienting effect that manifests as the performance difference between cued and uncued locations (valid vs. invalid trials); 2) an alerting effect that manifests as the performance difference between cued and uncued trials (with-cue vs. without-cue trials); and 3) an inhibition effect that manifests as a counteracting force to null the potential facilitation effect at the cued region - and as a persisting negative attentional bias against the previously cued region when the cue no longer appears (Lin \& Murray, 2015a). Table 2 summarizes the manifestations of the three attentional effects, the optimal conditions to observe them, and their relations to visual awareness.

It is common knowledge that a single exogenous cue can summon attention to the cued location, typically referred to as focal or selective attention, but less appreciated is the notion that a double cue also can direct multifocal or divided attention to the cued locations, ${ }^{3}$ by way of the same capture mechanism. Similarly, that a double cue can alert attention is assumed in traditional models of alerting, but less well recognized is that a single cue can also alert attention - an effect that is evident based on faster responses in cued than uncued trials.

\footnotetext{
${ }^{3}$ We consider directing multifocal attention from fixation to the two cued locations a form of orienting, in the same sense that directing attention away from fixation to one cued location is considered orienting - in both cases, attention is directed away from one location to elsewhere. Although not typically done in alerting research, it is a simple extension to create valid and invalid trials by expanding the set of relevant locations from just the two cued locations to including additional locations as well.
}

Above all, extent theories of attention generally give little thought to, or simply fail to anticipate, the potential inhibitory effect arising from exogenous cues; yet such inhibition - as is demonstrated here and elsewhere (Lin \& Murray, 2015a)can play a powerful role in attention by counteracting the facilitatory effect from cueing.

This three-component model reveals a nuanced picture of exogenous cueing. Under this model, it becomes necessary to consider how a seemingly simple manipulation of the cue might tap into orienting, alerting, and inhibition processes. Focusing on alerting, next we present our view on how alerting might be defined and studied, and how it is regulated.

What aspects of alerting does the ANT measure? The measure of orienting and alerting in the current study is couched within the attention network theory using the ANT — a widely adopted framework in cognitive, developmental, and clinical research of visual attention (Petersen \& Posner, 2012). There is good consensus that the ANT, by comparing performance between valid and invalid trials, taps into spatial orienting (specifically, focal or selective attention), but it is less clear just what aspects of alerting the ANT measures.

To gain a better understanding of this issue, it is necessary to define alerting first. Alerting is "achieving and maintaining a state of high sensitivity to incoming stimuli" (p. 7, Posner \& Rothbart, 2007). Based on the scope of the enhanced sensitivity, two types of alerting need to be distinguished. One concerns general arousal or alertness, which is closely related to levels of consciousness and distinguishes between such states as deep sleep, drowsiness, and full wake. The other type has to do with specific readiness to respond to a selective source of information, which is conceptually related to orienting ${ }^{4}$ and is exemplified by monitoring over space (e.g., left and right, as opposed to elsewhere), time (e.g., $\sim 1$ second later, as opposed to 5 seconds later), or modality (e.g., visual, as opposed to auditory). Frequently, it is the specific readiness - such as spatial monitoring in visual tasks - that is being studied in alerting research.

Posner (2008) suggests that the double cue in the ANT serves as a global warning signal to increase the speed of attention (or response) to the impending target. However, because in a typical ANT procedure only two locations are relevant, the double cue is likely to increase the specific readiness to respond to the two potential target locations (as opposed to other locations or a different modality). In other words, the effect of the double cue is "global" (left and right) only in comparison with the single cue (left or right).

\footnotetext{
${ }^{4}$ In such cases, what distinguishes orienting from alerting is that spatial orienting effects reflect a spatial shift - in particular, reorienting - of attention, as indexed by the difference between valid and invalid trials, whereas alerting effects reflect an enhancement in spatial monitoring, as indexed by the difference between cued trials (all locations being cued) and uncued trials (no location being cued).
} 
Table 2 Three types of attentional effects arising from an exogenous cue - such as a single cue or a double cue

\begin{tabular}{|c|c|c|c|}
\hline Effect & Manifestation & Optimal condition & Relation to awareness \\
\hline Orienting & $\begin{array}{l}\text { Capture of spatial attention to the cued } \\
\text { location and then reorienting of } \\
\text { attention to non-cued locations } \\
\text { (i.e., inhibition of return) }\end{array}$ & $\begin{array}{l}\text { When the CTOA is around } 100 \mathrm{~ms} \\
\text { (for the initial capture effect) or } \\
300 \mathrm{~ms} \text { and longer (for the } \\
\text { subsequent reorienting) }\end{array}$ & $\begin{array}{l}\text { Awareness of the cue } \\
\text { may not be necessary }\end{array}$ \\
\hline Alerting & $\begin{array}{l}\text { Enhancement in arousal and mental } \\
\text { preparation }\end{array}$ & $\begin{array}{l}\text { Within limits, when CTOA is long } \\
\text { and the cue is contextually } \\
\text { task-relevant }\end{array}$ & $\begin{array}{l}\text { Awareness of the cue } \\
\text { helps but may not be } \\
\text { necessary }\end{array}$ \\
\hline Inhibition (executive control) & $\begin{array}{l}\text { Inhibition of the cued location and a } \\
\text { global slowdown }\end{array}$ & $\begin{array}{l}\text { When the cue is spatial-temporally } \\
\text { close to the target, task-irrelevant, } \\
\text { distracting, and at a fixed location }\end{array}$ & $\begin{array}{l}\text { Awareness of the cue } \\
\text { may be necessary }\end{array}$ \\
\hline
\end{tabular}

CTOA, cue-target onset asynchrony

Although in the course of an experiment, some fluctuations of general vigilance are expected, when factoring in adaptation and habituation effects, it is not clear how strongly sensory cues may modulate trial-by-trial general arousal, which appears to be more sensitive to task difficulty and efforts (Kahneman, 1973).

If, as reasoned above, the double cue increases response readiness to the two cued locations, what would be responsible for the alerting effect? Because the double cue draws transient attention to the two spatial loci where the potential target is to appear, conceivably bottom-up divided attention ${ }^{5}$ is engaged to enhance processing at these loci (Bay \& Wyble, 2014; Sergent et al., 2013; Solomon, 2004; White, Lunau, \& Carrasco, 2014), leading to a behavioral alerting effect. However, the alerting effect in prior ANT research cannot always be interpreted as reflecting purely bottom-up processes. Although the double cue provides no spatial information for the target, its effect on alertness, as demonstrated here, is sensitive to the informativeness of the comingled single cues. By overlooking this potent top-down factor in determining the degree of specific response readiness, the alerting effect obtained in some prior research is likely to be partly driven by top-down expectation.

Two sources of phasic alerting What are the potential sources of phasic alerting? The present study shows that phasic alertness is enhanced by the contextual taskrelevance of the cue, and it can be evoked by an invisible, task-irrelevant cue. This observation highlights two distinct mechanisms in control of phasic alertness: one internal, based on top-down expectation; the other external, based on bottom-up stimulus properties. Therefore, we can become alert, heightening our response readiness while waiting for something to appear-such as

\footnotetext{
5 By “divided attention", we mean spatial attention that is being transiently distributed across multiple spatial locations. In prior studies, the term has also been used to refer to phenomena such as multi-tasking (e.g., driving and talking) and sustained attention to multiple channels of information (e.g., detecting targets from two auditory streams).
}

being more sensitive to the sound coming from the door when we are expecting a guest. Likewise, an external, imperative event - without necessarily engaging our awareness - can catch our attention and momentarily disengage us from our inner thoughts. As they alert us, these internal and external events typically intrude into our consciousness, although under certain circumstances, they also may escape our consciousness - in the form of unconscious alerting.

The current study provides insights into the intrinsic stimulus properties that may drive phasic alerting. In particular, Experiment 2 suggests that visual awareness of the stimulus is not required. In this regard, stimulus salience as defined in psychological terms - things that stand out conspicuously - also is not required, nor is it sufficient. Instead, things that stand out physically may be what elicit phasic alerting, such as the onset cue demonstrated in Experiment 2 (see Li, 2002). This possibility is consistent with a study using high-frequency, chromatic, flicker cues to elicit alerting and orienting - although in this case the attention effect was weaker when the flicker could not be perceived than when it could (Lu, Cai, Shen, Zhou, \& Han, 2012). In general, subliminal cues may enhance evidence accumulation and integration in the visual system and thus reduce the time needed to reach the decision-making threshold in a diffusion process (Smith \& Ratcliff, 2009).

Relations between phasic alerting and temporal orienting This dual-source account of phasic alerting builds on and extends previous theoretical dichotomy in spatial (Posner, 1980) and temporal (Lawrence \& Klein, 2013) attention. It is supported by the main observations reported in this study and may help to connect two seemingly distinct lines of research: attentional networks studied in the ANT (Callejas, Lupianez, \& Tudela, 2004; Fan et al., 2009; Fan et al., 2005; Fan et al., 2002) and temporal orienting as induced by cues that are indicative of when the target will appear (Coull, Frith, Buchel, \& Nobre, 2000; Coull \& Nobre, 1998; Niemi \& Naatanen, 1981). 
In the spatial domain, the orienting effect is characterized not so much by the initial orienting behavior-as argued above, both single and double cues involve directing attention away from the fixation to the cued region, with their difference being a matter of spatial extent- but more by the subsequent reorienting behavior. It is this reorienting behavior - attention being redirected from the originally cued location to an uncued location - that helps to distinguish orienting effects from alerting effects. Applying this definition to the temporal domain, temporal orienting can be characterized by reorienting of attention from the cued time point to an uncued time point. This definition brings out a conceptual difficulty with the term "temporal orienting," for although reorienting attention in space is a relatively intuitive and concrete actvisual search comes to mind-reorienting attention in time is more elusive. Consider a serial presentation of four items at the fixation where the target may appear at the second (and cued) or the fourth (and uncued) time point. When the target does not appear at the cued time point, it is not clear whether attention is actively shifted to the uncued time point or attention is merely heightened at the relevant time point as time passes, like when there is no temporal cue available. This challenge is perhaps a general problem when one moves from a concrete medium, such as space, to a more abstract medium, time - one can easily shift attention back and forth between points in space but not in time, which is fleeting and irreversible.

Therefore, without strong evidence for temporal reorienting of attention, the distinction between temporal orienting and temporal alerting is blurry. If the relevant distinction lies in the precision of the temporal informationmore precise temporal information afforded by orienting cues than by alerting cues (Weinbach \& Henik, 2012) - then the difference appears to be a difference in the weightings to the time points: uneven weights in orienting (e.g., higher weight to the second time point and lower to the fourth time point) versus relatively even weights in alerting. Does this mean that alerting and temporal orienting are parallel and distinct (Weinbach \& Henik, 2012)? We think not; in such cases, we believe that temporal orienting has much to do with internal, top-down alerting. A difference in weightings may simply reflect a difference in the specific top-down choice. As an analogy, when the guest is likely to arrive through either the front door or the back door, one becomes alert to sensory inputs coming from both doors; but when the front door is the only appropriate option, one becomes selectively tuned to the front door.

The ostensibly parallel and distinct relationship between alerting and temporal orienting is drawn based not on their respective underlying neural mechanisms but on the behavioral tests that induce them: alerting as induced by supposedly task-irrelevant cues, and temporal orienting, by task-relevant cues (Weinbach \& Henik, 2012). However, this procedure- based classification of phenomena may be inadequate, not least because, as Experiment 1 shows, in traditional studies of visual alerting the supposedly task-irrelevant cues actually may be task-relevant, which casts some doubt on the relevance of task relevance in this debate. Furthermore, presupposing or restricting temporal alerting to be induced only by task-irrelevant cues also fails to account for its internal, top-down source.

Neurophysiological recordings in animals provide further evidence for a common mechanism. Exogenous cues are generally found to trigger phasic responses in locus coeruleus (LC) neurons and stimulate the release of norepinephrine (NE), which spreads to task-relevant cortical circuits to enhance neural sensitivity and boost performance (Aston-Jones \& Bloom, 1981; Foote, Astonjones, \& Bloom, 1980). It also has been show that an awaited task-relevant cue, the kind that induces temporal orienting, transiently activates the LC-NE system in a fashion that is similar to exogenous cues (AstonJones, Rajkowski, Kubiak, \& Alexinsky, 1994; Bouret \& Richmond, 2009). The LC-NE system, in other words, is responsive to events that elicit an alerting response, whether they are external or internal (Sara \& Bouret, 2012). These observations are inconsistent with the assumption that temporal orienting is distinct from alerting (Weinbach \& Henik, 2012), but they agree with the proposal that temporal orienting is driven by the internal source of alerting. From the perspective of the gating theory of attention (Olivers \& Meeter, 2008; Reeves \& Sperling, 1986), the detection of a relevant cue and an exogenous cue may both be thought of to open a transient attention gate, allowing entry into short-term memory.

\section{Relations between phasic alerting and attentional capture} We have argued that the external source of phasic alerting is based on bottom-up, stimulus-driven attentional capture. Attentional capture is an active topic on its own, and many studies have been conducted to investigate when a taskirrelevant object captures attention (as reviewed in Theeuwes, 2010). How are these studies related to alerting? And what can we learn about alerting from attentional capture?

We suggest that studies on the two topics differ in three main aspects, but these differences appear to be mostly procedural. The first difference is the scope of attentional focus. Traditional studies in attentional capture emphasize attention being drawn to one spatial location (by a singleton), whereas studies in phasic alertness emphasize attention to multiple locations (by a double cue). This difference, as argued previously, reflects not so much a difference in kind but a difference in the scope of attentional distribution. The second difference is the spatial uncertainty of the target. Studies in attentional capture typically use a visual search task that involves multiple potential target locations (four or more), whereas studies in phasic alerting typically use a simple detection or 
discrimination task that involves two potential target locations. The third difference has to do with performance cost and benefit. Studies in attentional capture emphasize performance cost as a result of attention being drawn to a distractor, whereas studies in phasic alerting focus on performance benefit as a result of attention drawn to the locations where the target may potentially appear. Performance cost and benefit likely reflect two sides of the same coin: drawing attention to the distractor location impairs performance, whereas drawing attention to the target location benefits performance.

The above analysis suggests that studies in attentional capture and alerting are more related than they appear. Indeed, to the extent that salient events can capture attention to alert us, attentional capture can be thought of as a process that underlies the external control of phasic alertness (in addition to the potential orienting and inhibition effects discussed previously). In attentional capture, it is often debated whether a salient item can capture attention independently of the observer's goal (Bacon \& Egeth, 1994; Folk \& Remington, 1998; Theeuwes, 2010). It has been suggested that a salient distractor may automatically trigger an attentional capture signal, but this signal can be suppressed to prevent an attentional capture behavior (Sawaki \& Luck, 2010), providing partial support for both positions. This debate raises an interesting question for alerting, namely whether external control of phasic alertness operates independently of internal control. Can an abrupt-onset cue alert attention independently of top-down expectation?

In the current study, it seems unlikely that participants were actively expecting to see the cue when it was masked from awareness. Nevertheless, it is possible that task set-the configuration of attention to detect the presence or onset of a target dot—could potentially sensitize cue processing, thereby contributing to the alerting effect. In studies where a brief tone is used to alert attention-a procedure known as ANT Interaction, or ANT-I for short (Callejas, Lupianez, Funes, \& Tudela, 2005; Callejas et al., 2004) - it is likely that the tone captures attention to allow observers to prepare and focus topdown attention on the upcoming target locations. In such cases, salient cues appear to trigger a cascade of bottom-up and top-down attention.

Independence - and interdependence - of orienting and alerting We have revealed the connections between alerting and temporal orienting and attentional capture. We now turn to the relationship between alerting and spatial orienting, a central question in the attention network theory-which is also the main theoretical backdrop of the present study. According to this theory, conscious attention comprises three networks - alerting, spatial orienting, and executive controlthat are relatively independent of each other (Petersen \& Posner, 2012). Evidence for their independence has come from the ANT, which has shown weak correlations between measures of the three components (Fan et al., 2002). The current study further suggests that unconscious alerting and unconscious orienting appear to be largely uncorrelated with each other, providing preliminary evidence for independent attentional networks in unconscious attention. In addition, conscious attention and unconscious attention appear to be uncorrelated, suggesting nonoverlapping neural mechanisms.

However, the observation that manipulating the task relevance of the single cue can modulate the alerting effect reveals their interdependence - an underappreciated aspect of attentional networks (Callejas et al., 2005; Callejas et al., 2004; Fan et al., 2009). Although the attention network theory emphasizes the independence between orienting and alerting, their interdependence as components in a complex system - the brain-should not come as a surprise. As an analogy, consider two persons riding on a boat. Although the two can behave relatively independently — one may stand up and stretch his arms, the other sit and stretch his legs - they are fundamentally constrained by being in the same boat: rocking the boat by one person will affect the movement of the other person. Similarly, when the single cue is predictive of the target location, cues as a category gain top-down priority, to affect the impact of the double cue. The effects of orienting and alerting can still be largely uncorrelated, because they reflect different behaviors: orienting as a spatial shift of attention, alerting as multifocal attention to the two cued locations.

Measuring attention in applied and clinical settings So far, we have focused on the theoretical implications. Given the importance of attention in supporting everyday activity and its prevalent deficits in brain disorders, a better understanding of the measurement of attention is crucial. The ANT is a popular behavioral tool to measure attention in applied and clinical settings. It has been suggested that the alerting function may be sensitive to normal aging and attention deficit disorder (Posner \& Rothbart, 2007), and orienting, alerting and executive functions may be disrupted in autism (Keehn, Muller, \& Townsend, 2013). However, several challenges limit the potential utility of the ANT in these applications.

In particular, the underlying theory, the attention network theory, relies on accepting a null hypothesis-a lack of correlations between measures of attentional components in the ANT. A lack of correlations, however, may be due to factors unrelated to the existence of independent attention networks. For example, it may be due to unreliable measures of the variables. The reliability of measurement places an upper bound on the observable correlation; mathematically, assuming random measurement error, the maximal correlation of two variables is the square root of the reliabilities with which they are measured (Nunnally, 1970). It may also be due to different strategies being used in different types of trials. For example, it is possible that "participants might respond differently to the conditions 
used to calculate the difference scores in ways that are not directly tied to the type of attention that score is assumed to represent" (p. 720, Redick \& Engle, 2006).

An advantage of using invisible cues is that it may minimize potential differences in the strategy used in different conditions - the cue is after all invisible. If so, the subliminal ANT method (S-ANT) holds promise as a better tool for isolating components of attention by minimizing the effect of top-down strategy. This potential methodological improvement may be of practical significance, considering that the utility of the ANT in developmental, clinical, and applied research rests on it being a reliable measure (Ishigami \& Klein, 2010; MacLeod et al., 2010; Wang et al., 2015), and differing strategies across conditions can corrupt the reliability of the ANT (Redick \& Engle, 2006; Rueda et al., 2004).

In general, the ANT has the advantage of measuring alerting, orienting, and executive attention within a 30-min session, lending it favorably to potential clinical applications. However, this efficiency comes with some significant costs. For example, although the orienting effect is typically calculated by collapsing across different executive attention conditions (i.e., congruent, incongruent, and neutral conditions), the correlations of orienting effects between the three conditions are rather weak; the same is true for alerting and executive attention in the different orienting conditions (McConnell \& Shore, 2011). Moreover, splithalf and test-retest reliabilities are low for alerting and orienting effects and moderately high for executive attention. Reliabilities are only slightly improved in the ANT-I, where the double cue is not used and instead in half of the trials a tone, serving as the alerting cue, precedes the single cue (Ishigami \& Klein, 2010). Reliabilities may also be slightly improved in the original ANT but at the cost of additional trials (Fan et al., 2009).

At the conceptual level, how much we can improve measurement reliability is constrained by two factors: the degree of intraindividual variability in the attentional components and the precision of the measurement tools. Tackling the problem of intraindividual variability requires us to control for brain states during testing. Behavioral performance tends to be susceptible to the influence of brain states, such as motivation and mind wondering. Therefore, controlling the aspects of the testing environment (e.g., room layout, lightening, and temperature) that might affect brain states would be helpful. Controlling internal states is more difficult, but attempts should be made to ensure that the instruction is clear and easy to follow, the participant's strategy constant, and their motivation and energy level appropriately high throughout the experiment. It would also be useful to gain access to their subjective states in each block by querying participants, such as: How motivated were you during the task? How hard did you try in the task? Information about subjective states can then be quantified and incorporated in performance evaluation - such as for quality control purpose and for discounting performance.

Improving the precision of the tool to measure attention depends on improving existing tools and devising novel ones. In general, RT measurement is noisy (Salthouse \& Berish, 2005), but data quality may be improved through thoughtful experimentation, such as behavioral training (e.g., training proper eye fixation if stable fixation is important; training fast responses if RT is crucial) and proper performance feedback (e.g., reminding the participant to focus on the task when an incorrect response is given to a very easy trial, or an impossibly fast response is given in response to the target). Moreover, RT measurement may be complemented by other measures. For example, an important goal is to discover stable brain structures and connectivity that can reliably index the mental function of interest, such as orienting and alerting (Rosenberg et al., 2016); the measure of relevant brain structures and connectivity patterns would then prove useful.

\section{Conclusions}

Despite its central role in regulating cognition and performance, alerting remains an understudied dimension of attention and consciousness, with its operations poorly understood (Raz \& Buhle, 2006). In this study, we identified a crucial role of contextual relevance - the informativeness of the single cue that is being mixed with the double cue - in determining the behavioral effect of the double cue, which reveals a new source of top-down influence on alerting. Furthermore, we uncovered a bottom-up form of alerting effect that can occur before the cue enters our awareness; this unconscious effect can manifest itself where a conscious effect could not-an intriguing contrast explained by awareness-induced inhibition (Lin \& Murray, 2015a).

These empirical observations lend support to the theoretical position that a simple visual cue can engage three components of attention-orienting, alerting, and inhibition-to determine the behavioral cueing effect. We showed that alerting can be defined and studied as general arousal and specific response readiness, and it is regulated by both internal, top-down expectation and external, bottom-up stimulus properties. We revealed the strong connections of alerting to temporal orienting, attentional capture, and spatial orienting. Ultimately, progress in attention research has the potential to transform clinical applications, and an important goal is to improve the measurement of attention, such as by controlling the degree of intraindividual variability in the attentional components and by improving the precision of the measurement tools. 


\section{Appendix 1}

\section{Speed-accuracy tradeoff analyses in Experiment 1}

When there is a speed-accuracy tradeoff, faster responses can be attributed to the observer adopting a more liberal criterion rather than to faster processing, and vice versa. That increasing CTOA reduced both false-alarm rates and RT provides clear evidence against speed-accuracy tradeoffs, which would predict a reduction in RT as resulting from an increase in false-alarm rates. (False-alarm rates were used as a proxy for accuracy, because errors were primarily false alarms, with relatively few misses; Table 1). To directly examine speed-accuracy tradeoffs, below we turned to integrative measures of hit and false-alarm rates: first focusing on criterion $(c)$, calculated as: $-(z$ (hit $)+z($ false alarm $)) / 2$; and then on sensitivity $\left(d^{\prime}\right)$, calculated as: $z$ (hit $)-z$ (false alarm).

We first examined whether the crucial RT effects were due to shifts in criterion. For orienting, we found no evidence that participants adopted a more biased, liberal criterion in the valid than invalid trials at the shortest CTOA (when facilitation was found), nor evidence for the reverse pattern at the longer CTOAs (when inhibition was found). An ANOVA with the factors CTOA $(16.7 \mathrm{~ms}, 316.7 \mathrm{~ms}$, vs. $616.7 \mathrm{~ms}$ ), validity (valid cue vs. invalid cue), and group (uninformative-cue group vs. informative-cue group) on $c$ revealed only a main effect of CTOA $(F(2,76)=16.47, p<$ $\left.0.001, \eta_{p}^{2}=0.30\right)$, without a main effect of validity $(F(1$, $\left.38)=2.22, p=0.144, \eta_{p}^{2}=0.06\right)$ or their interaction $(F(2$, 76) $\left.=0.82, p=0.443, \eta_{p}^{2}=0.02\right)$. The CTOA main effect indicated that when the CTOA was lengthened, the absolute value of $c$ decreased (i.e., less biased; linear trend: $F(1$, $38)=25.38, p<0.001, \eta_{p}^{2}=0.40$; Table 1)-with a corresponding reduction in overall RT (Fig. 2, left). This relationship echoes the results from false-alarm rates above, and together they provide clear evidence against speedaccuracy tradeoffs.

A similar pattern was observed in alerting effects. Because of cue-induced false alarms in double-cue but not in no-cue trials, the double-cue trials were more biased than the no-cue trials, particularly at shorter CTOAs. Importantly, however, at the shortest CTOA, despite double-cue trials being more biased than no-cue trials, there was no corresponding RT alerting effect; yet at the longest CTOA, where the criterion was comparable between double-cue trials and no-cue trials, there was nevertheless a strong RT alerting effect (Table 1). Specifically, an ANOVA with the factors CTOA $(16.7 \mathrm{~ms}, 316.7 \mathrm{~ms}$, vs. $616.7 \mathrm{~ms}$ ), cue (no cue vs. double cue), and group (uninformative-cue group vs. informative-cue group) on $c$ revealed a main effect of CTOA $(F(2,76)=7.17, p=0.001$, $\left.\eta_{p}^{2}=0.16\right)$, a main effect of cue $(F(1,38)=11.79, p=0.001$, $\left.\eta_{p}^{2}=0.24\right)$, and their interaction $(F(2,76)=5.97, p=0.004$, $\left.\eta_{p}^{2}=0.14\right)$. The CTOA and cue interaction effect reflected that, due to cue-induced false alarms, $c$ was more biased in double-cue trials than in no-cue trials when the CTOAs were $16.7 \mathrm{~ms}\left(F(1,38)=15.52, p<0.001, \eta_{p}^{2}=0.29\right)$ and $316.7 \mathrm{~ms}$ $\left(F(1,38)=7.47, p=0.009, \eta_{p}^{2}=0.16\right)$, but $c$ was comparable when the CTOA was $616.7 \mathrm{~ms}(F(1,38)=0.72, p=0.401$, $\left.\eta_{p}^{2}=0.02\right)$.

To further examine speed-accuracy tradeoffs, we next used $d^{\prime}$ as a measure of accuracy. Although $d^{\prime}$ tends to amplify small differences when hit rates are at ceiling (as is our case; Table 1), nevertheless it affords us to visualize speed-accuracy tradeoffs across conditions. Figure 5 shows the scatterplots of $d^{\prime}$ and RT from each individual participant in the uninformative-cue group; Fig. 6, the informative-cue group.

For orienting effects, in the uninformative-cue group (Fig. 5, left), neither did facilitation at CTOA of $16.7 \mathrm{~ms}$ nor did inhibition at CTOAs of $316.7 \mathrm{~ms}$ and $616.7 \mathrm{~ms}$ come with a significant difference in $d^{\prime}$ between valid and invalid trials $(16.7 \mathrm{~ms}: t(19)=0.94, p=0.361, d=0.21 ; 316.7 \mathrm{~ms}: t(19)=$ $-1.88, p=0.075, d=-0.42$, a trend opposite to speed-accuracy tradeoff; $616.7 \mathrm{~ms}: t(19)=-0.30, p=0.769, d=-0.07$ ). The same pattern was observed in the informative-cue group (Fig. 6, left; 16.7 ms: $t(19)=-0.38, p=0.707, d=-0.09$; $316.7 \mathrm{~ms}: t(19)=-0.68, p=0.503, d=-0.15 ; 616.7 \mathrm{~ms}: t(19)$ $=-0.60, p=0.558, d=-0.13$ ).

For alerting effects, in the uninformative-cue group (Fig. 5, right), at CTOA of $316.7 \mathrm{~ms}$, the significant alerting effect was not accompanied by different $d$ 's between double-cue trials and no-cue trials $(t(19)=-1.67, p$ $=0.111, d=-0.37$ ); conversely, at CTOAs of $16.7 \mathrm{~ms}$ and $616.7 \mathrm{~ms}$, there was no alerting effect, despite significantly lower $d$ 's in double-cue trials than in no-cue trials (16.7 ms: $t(19)=-3.07, p=0.006, d=-0.69$; $616.7 \mathrm{~ms}: t(19)=-2.18, p=0.042, d=-0.49)$. And in no case did differences in $d^{\prime}$ across participants correlate with the orienting or alerting effect (Fig. 5). A similar pattern was observed in the informative-cue group (Fig. 6, right). Specifically, the significant alerting effects at CTOAs of $316.7 \mathrm{~ms}$ and $616.7 \mathrm{~ms}$ were not accompanied by different $d$ 's between double-cue trials and no-cue trials $(316.7 \mathrm{~ms}: t(19)=-1.41, p=0.174, d=-0.32$; 616.7 ms: $t(19)=-0.95, p=0.354, d=-0.21)$; conversely, at CTOA of $16.7 \mathrm{~ms}$, there was no alerting effect, despite a significantly lower $d^{\prime}$ in double-cue trials than in no-cue trials $(t(19)=-4.52, p<0.001, d=$ $-1.01)$. Again, in no case did differences in $d^{\prime}$ across participants correlate with the orienting or alerting effect (Fig. 6). Together, these results from $d^{\prime}$ and $c$ provide strong evidence that speed-accuracy tradeoffs could not account for the main findings reported. 


\section{Orienting}

Uninformative-cue group
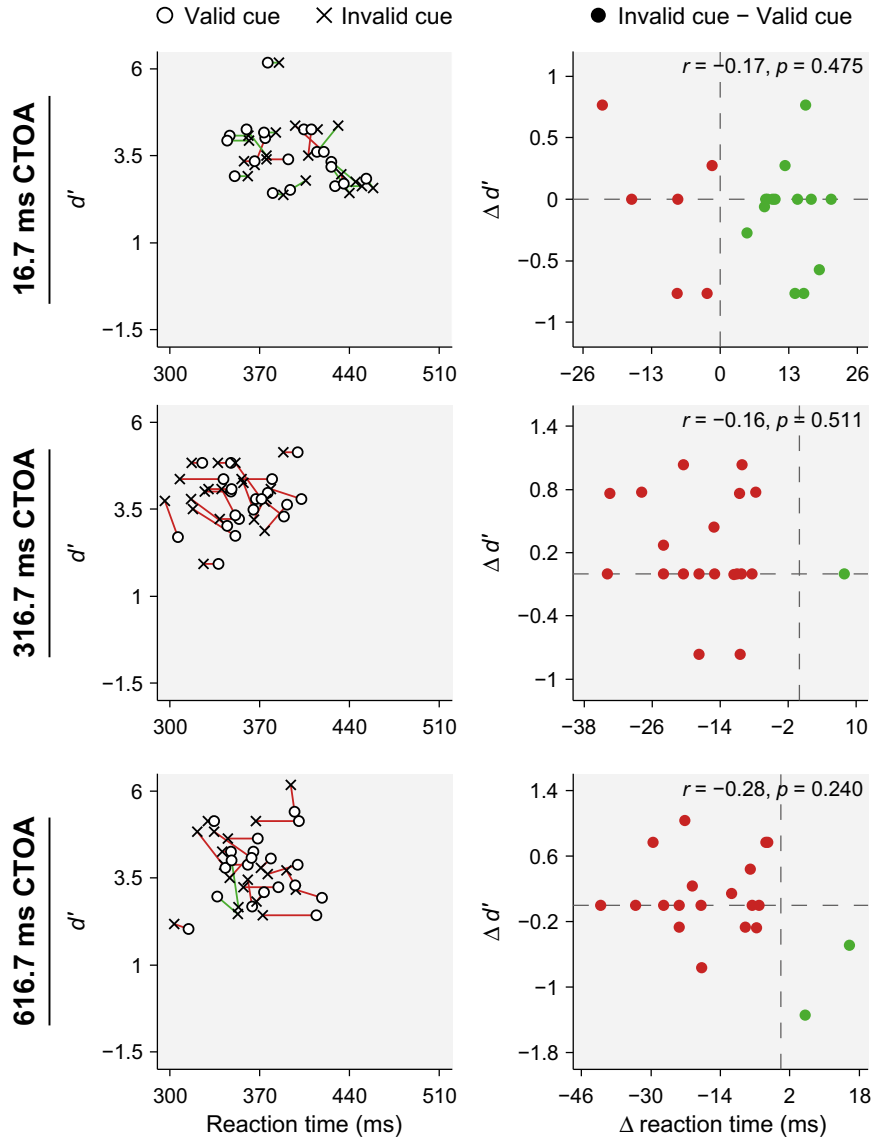

Fig. 5 Scatterplots of $d^{\prime}$ (a measure of accuracy) and reaction time for each individual participant from the uninformative-cue group in Experiment 1. Each line connects two data points from the same participant. Green lines and dots represent positive orienting or alerting effects; red lines and dots, negative effects. To facilitate comparisons

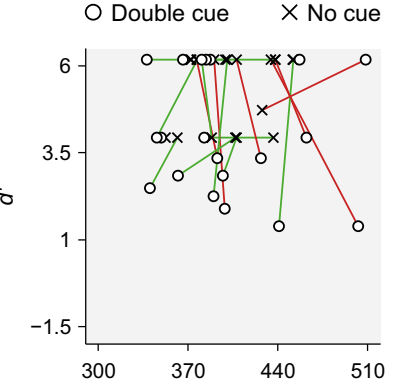

Alerting
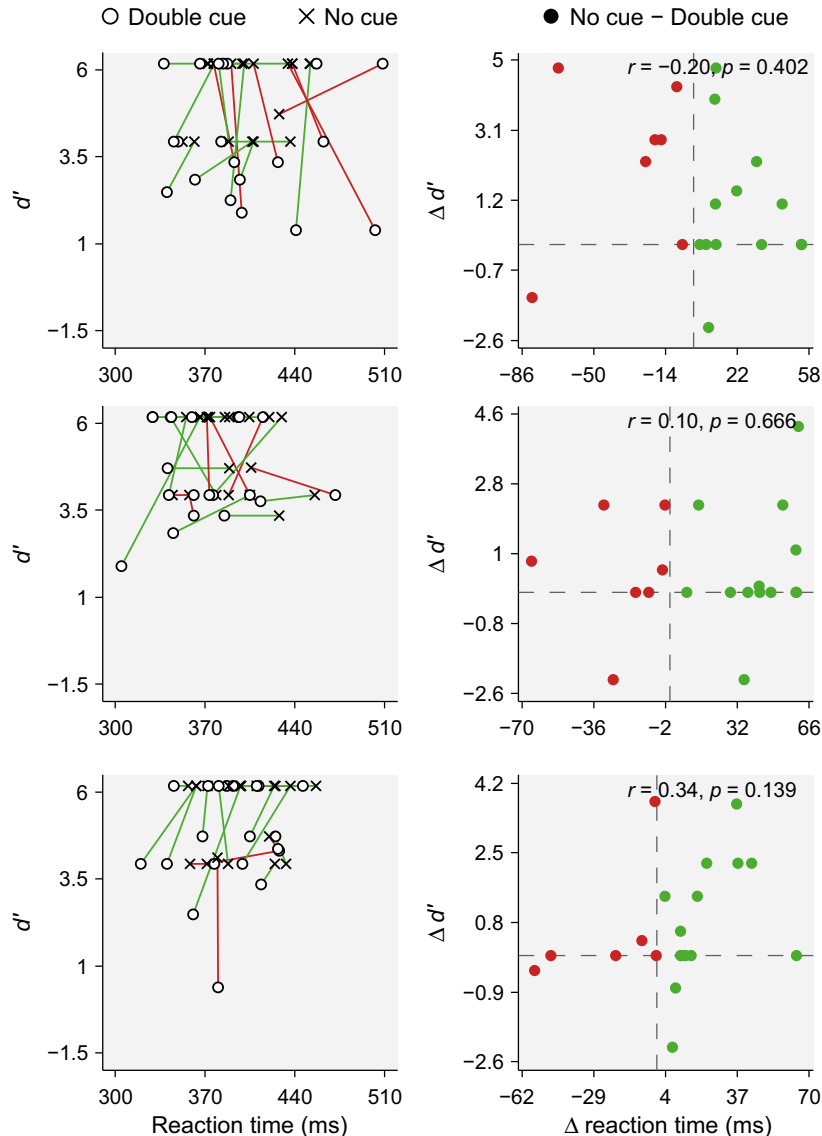

across conditions, the panels in columns 1 and 3 -as well as those in Fig. 6- have the same scale, but due to large individual differences across conditions, the panels in columns 2 and 4 have different scales to allow better visualization of individual data points. CTOA, cue-target onset asynchrony 


\section{Orienting}

O Valid cue $\quad \times$ Invalid cue
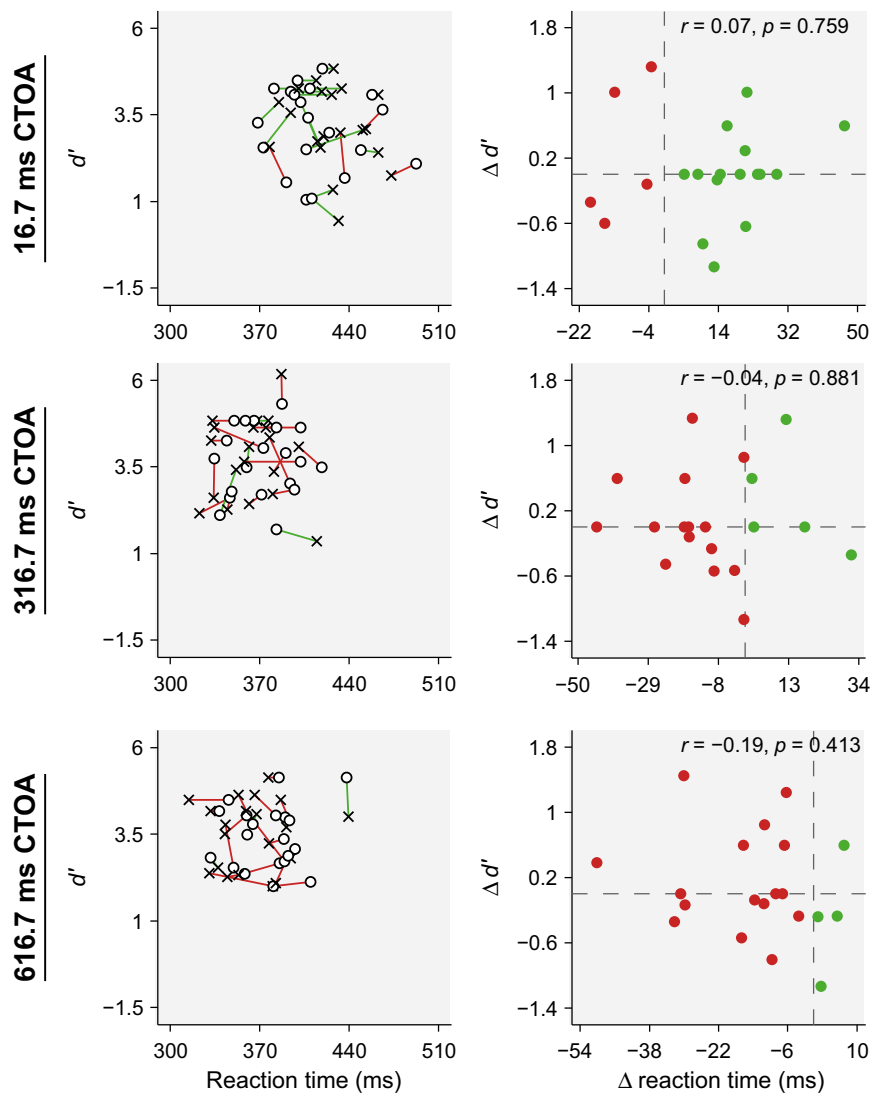

Fig. 6 Scatterplots of $d^{\prime}$ (a measure of accuracy) and reaction time for each individual participant from the informative-cue group in Experiment 1. Each line connects two data points from the same participant. Green lines and dots represent positive orienting or alerting effects; red lines and dots, negative effects. To facilitate comparisons

\section{Alerting}

O Double cue $\times$ No cue
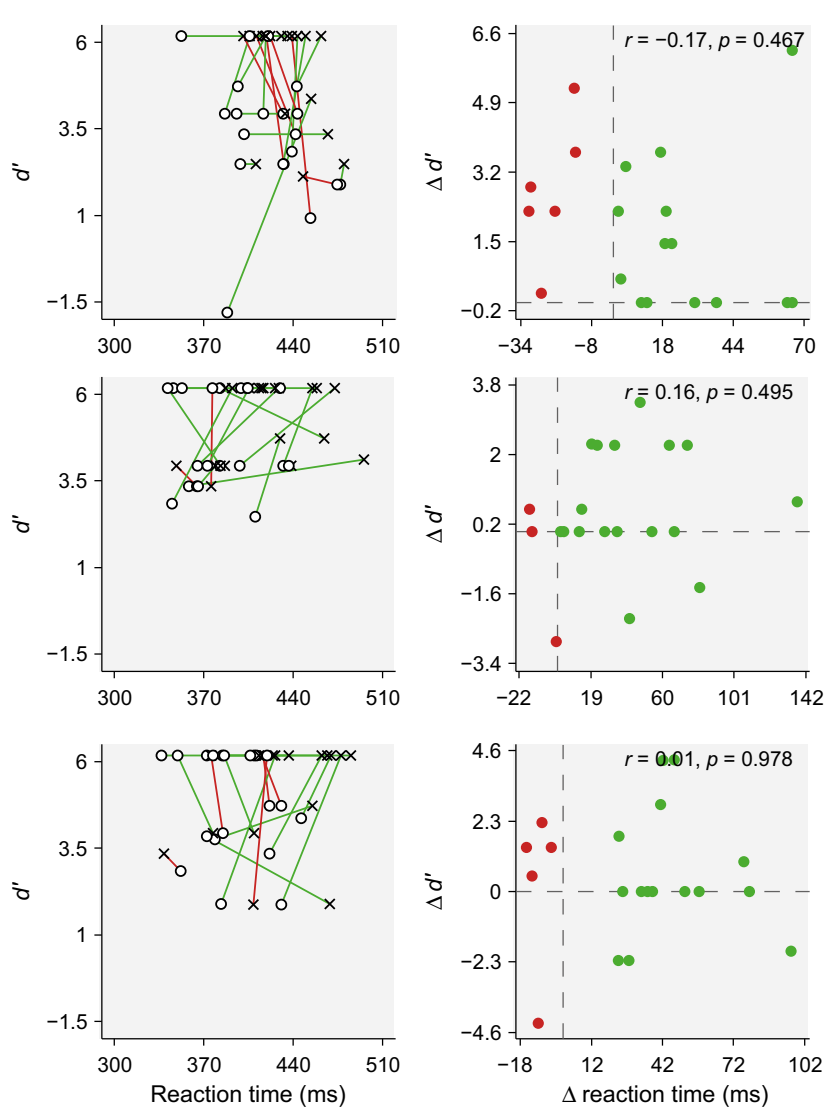

across conditions, the panels in columns 1 and 3 -as well as those in Fig. 5- have the same scale, but due to large individual differences across conditions, the panels in columns 2 and 4 have different scales to allow better visualization of individual data points. CTOA, cue-target onset asynchrony 


\section{Appendix 2}

\section{Speed-accuracy tradeoffs in Experiment 2}

Similar to the effect of CTOA (Experiment 1), masking the cue from awareness (Experiment 2) reduced both false alarm rates and RT, providing clear evidence against speed-accuracy tradeoffs, which would predict a reduction in RT with a corresponding increase in false-alarm rates.

To further examine speed-accuracy tradeoffs, we first looked at criterion. As in Experiment 1, for orienting effects, analysis on $c$ and RT showed no evidence of speed-accuracy tradeoffs. If anything, for the facilitation effect from invisible cues, the criterion was actually less biased (more conservative) in valid than invalid trials (Table 1; see the Supplemental Online Material $\S 3$ for statistical results). For the alerting effect from invisible cues, there was no significant difference in criterion between double-cue trials and no-cue trials. On the other hand, for visible cues, despite being more biased in double-cue trials than in no-cue trials, this difference in bias did not translate into an alerting effect (Table 1; see the Supplemental Online Materials $\S 3$ for statistical results). In other words, alerting effects emerged without a criterion difference between double-cue trials and no-cue trials (when the cue was invisible); and conversely, being more biased did not necessarily confer a RT advantage in double-cue trials (when the cue was visible).

Next, we turned to sensitivity. Figure 7 shows the scatterplots of $d^{\prime}$ and RT from each individual participant. For orienting effects, we found that the majority of participants (13 of 18 in visible cues; 12 of 18 in invisible cues) showed no difference in $d^{\prime}$-and all of these participants showed a positive orienting effect. For alerting effects, invisible cues elicited an alerting effect without a corresponding difference in $d^{\prime}$ between double-cue and no-cue trials $(t(17)=$ $-0.95, p=0.365, d=-0.22)$; in contrast, visible cues did not elicit an alerting effect, despite a lower $d^{\prime}$ in double-cue trials than in no-cue trials $(t(17)=-1.64, p<0.001, d=-1.65)$ reflecting cue-induced false alarms. Regardless of cue visibility, differences in $d^{\prime}$ across participants did not correlate with the orienting or alerting effect (Fig. 7).

Taken as a whole, these results - concurrent reductions in false-alarm rates and RT by masking the cue from awareness; no differences in $c$ or $d^{\prime}$ when orienting and alerting effects were found; differences in $c$ or $d^{\prime}$ when orienting and alerting effects were not found; and no correlations between differences in $d^{\prime}$ across participants and the corresponding orienting or alerting effects - are consistent with Experiment 1 and provide strong evidence that speed-accuracy tradeoffs could not account for the main findings.
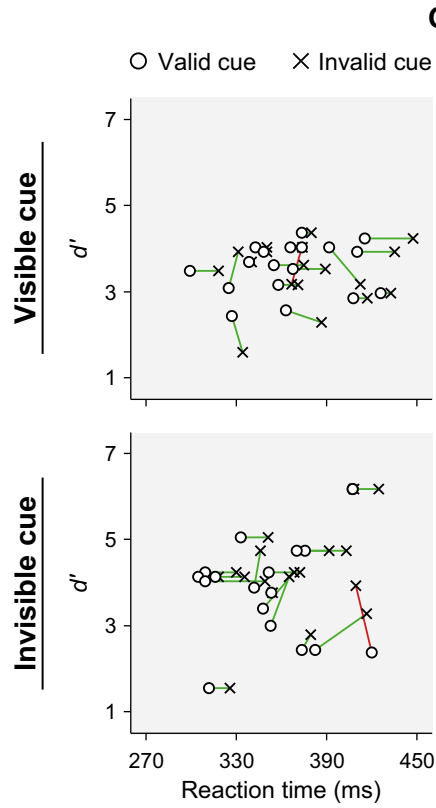

\section{Orienting}
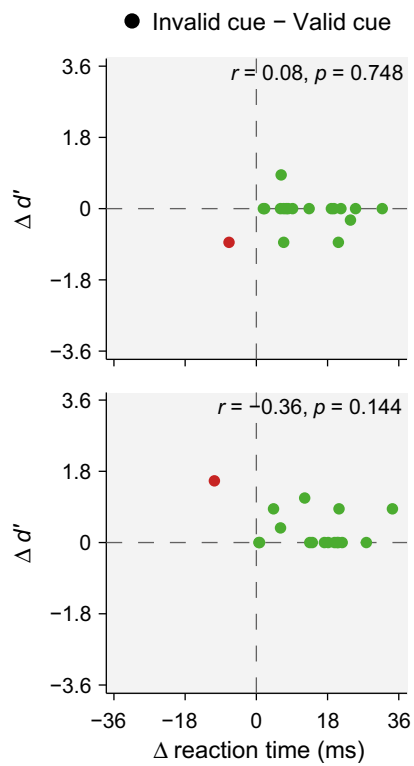

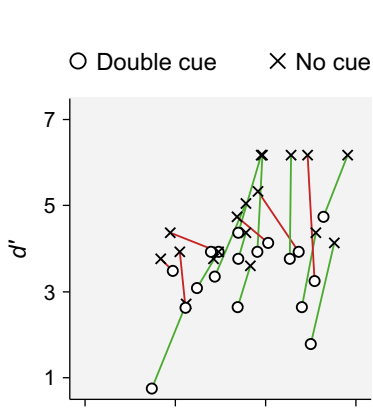

Alerting

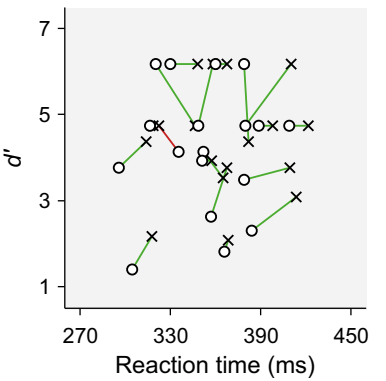

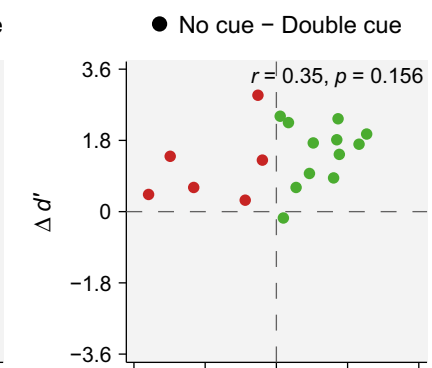

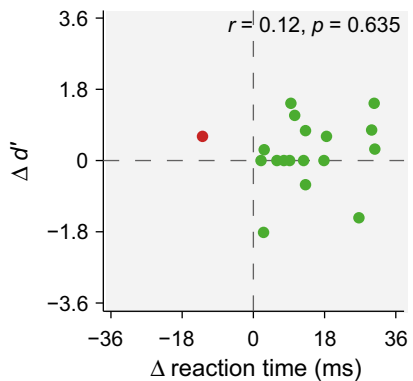

Fig. 7 Scatterplots of $d^{\prime}$ (a measure of accuracy) and reaction time for each individual participant in Experiment 2. Each line connects two data points from the same participant. Green lines and dots represent positive orienting or alerting effects; red lines and dots, negative effects 


\section{References}

Aston-Jones, G., \& Bloom, F. E. (1981). Norepinephrine-containing locus coeruleus neurons in behaving rats exhibit pronounced responses to non-noxious environmental stimuli. Journal of Neuroscience, 1(8), 887-900.

Aston-Jones, G., \& Cohen, J. D. (2005). An integrative theory of locus coeruleus-norepinephrine function: Adaptive gain and optimal performance. Annual Review of Neuroscience, 28, 403-450.

Aston-Jones, G., Rajkowski, J., Kubiak, P., \& Alexinsky, T. (1994). Locus coeruleus neurons in monkey are selectively activated by attended cues in a vigilance task. Journal of Neuroscience, 14(7), $4467-4480$

Bacon, W. F., \& Egeth, H. E. (1994). Overriding stimulus-driven attentional capture. Perception \& Psychophysics, 55(5), 485-496.

Bay, M., \& Wyble, B. (2014). The benefit of attention is not diminished when distributed over two simultaneous cues. Attention, Perception, \& Psychophysics, 76(5), 1287-1297.

Bouret, S., \& Richmond, B. J. (2009). Relation of locus coeruleus neurons in monkeys to Pavlovian and operant behaviors. Journal of Neurophysiology, 101(2), 898-911.

Callejas, A., Lupianez, J., Funes, M. J., \& Tudela, P. (2005). Modulations among the alerting, orienting and executive control networks. Experimental Brain Research, 167(1), 27-37.

Callejas, A., Lupianez, J., \& Tudela, P. (2004). The three attentional networks: On their independence and interactions. Brain and Cognition, 54(3), 225-227.

Cheal, M., \& Lyon, D. R. (1991). Central and peripheral precuing of forced-choice discrimination. Quarterly Journal of Experimental Psychology. A, Human Experimental Psychology, 43(4), 859-880.

Coull, J. T., Frith, C. D., Buchel, C., \& Nobre, A. C. (2000). Orienting attention in time: Behavioural and neuroanatomical distinction between exogenous and endogenous shifts. Neuropsychologia, 38(6), 808-819.

Coull, J. T., \& Nobre, A. C. (1998). Where and when to pay attention: The neural systems for directing attention to spatial locations and to time intervals as revealed by both PET and fMRI. Journal of Neuroscience, 18(18), 7426-7435.

Eckstein, M. P., Shimozaki, S. S., \& Abbey, C. K. (2002). The footprints of visual attention in the Posner cueing paradigm revealed by classification images. Journal of Vision, 2(1), 25-45.

Fan, J., Gu, X., Guise, K. G., Liu, X., Fossella, J., Wang, H., \& Posner, M. I. (2009). Testing the behavioral interaction and integration of attentional networks. Brain and Cognition, 70(2), 209-220.

Fan, J., McCandliss, B. D., Fossella, J., Flombaum, J. I., \& Posner, M. I. (2005). The activation of attentional networks. NeuroImage, 26(2), 471-479.

Fan, J., McCandliss, B. D., Sommer, T., Raz, A., \& Posner, M. I. (2002). Testing the efficiency and independence of attentional networks. Journal of Cognitive Neuroscience, 14(3), 340-347.

Fernandez-Duque, D., \& Posner, M. I. (1997). Relating the mechanisms of orienting and alerting. Neuropsychologia, 35(4), 477-486.

Folk, C. L., \& Remington, R. (1998). Selectivity in distraction by irrelevant featural singletons: Evidence for two forms of attentional capture. Journal of Experimental Psychology: Human Perception and Performance, 24(3), 847-858.

Foote, S. L., Astonjones, G., \& Bloom, F. E. (1980). Impulse activity of locus coeruleus neurons in awake rats and monkeys is a function of sensory stimulation and arousal. Proceedings of the National
Academy of Sciences of the United States of America, 77(5), 3033-3037.

Fuentes, L. J., \& Campoy, G. (2008). The time course of alerting effect over orienting in the attention network test. Experimental Brain Research, 185(4), 667-672.

Greene, D. J., Barnea, A., Herzberg, K., Rassis, A., Neta, M., Raz, A., \& Zaidel, E. (2008). Measuring attention in the hemispheres: The lateralized attention network test (LANT). Brain and Cognition, 66(1), 21-31.

Guzman-Martinez, E., Leung, P., Franconeri, S., Grabowecky, M., \& Suzuki, S. (2009). Rapid eye-fixation training without eyetracking. Psychonomic Bulletin \& Review, 16(3), 491-496.

Ishigami, Y., \& Klein, R. M. (2010). Repeated measurement of the components of attention using two versions of the Attention Network Test (ANT): Stability, isolability, robustness, and reliability. Journal of Neuroscience Methods, 190(1), 117-128.

Kahneman, D. (1973). Attention and effort. Englewood Cliffs, NJ: Prentice-Hall.

Keehn, B., Muller, R. A., \& Townsend, J. (2013). Atypical attentional networks and the emergence of autism. Neuroscience and Biobehavioral Reviews, 37(2), 164-183.

Klein, R. M. (2000). Inhibition of return. Trends in Cognitive Sciences, $4(4), 138-147$.

Lawrence, M. A., \& Klein, R. M. (2013). Isolating exogenous and endogenous modes of temporal attention. Journal of Experimental Psychology: General, 142(2), 560-572.

Li, Z. (2002). A saliency map in primary visual cortex. Trends in Cognitive Sciences, 6(1), 9-16.

Lin, Z., \& He, S. (2009). Seeing the invisible: The scope and limits of unconscious processing in binocular rivalry. Progress in Neurobiology, 87(4), 195-211.

Lin, Z., Lu, Z. L., \& He, S. (2016). Decomposing experience-driven attention: Opposite attentional effects of previously predictive cues. Attention, Perception, \& Psychophysics.

Lin, Z., \& Murray, S. O. (2013). Visible propagation from invisible exogenous cueing. Journal of Vision, 13(11), 1-15.

Lin, Z., \& Murray, S. O. (2014). Priming of awareness or how not to measure visual awareness. Journal of Vision, 14(1), 1-17.

Lin, Z., \& Murray, S. O. (2015a). More power to the unconscious: Conscious, but not unconscious, exogenous attention requires location variation. Psychological Science, 26(2), 221-230.

Lin, Z., \& Murray, S. O. (2015b). Automaticity of unconscious response inhibition: Comment on Chiu and Aron (2014). Journal of Experimental Psychology: General, 144(1), 244-254.

Lu, S. N., Cai, Y. C., Shen, M. W., Zhou, Y., \& Han, S. H. (2012). Alerting and orienting of attention without visual awareness. Consciousness and Cognition, 21(2), 928-938.

Lu, Z. L., \& Dosher, B. A. (1998). External noise distinguishes attention mechanisms. Vision Research, 38(9), 1183-1198.

MacLeod, J. W., Lawrence, M. A., McConnell, M. M., Eskes, G. A., Klein, R. M., \& Shore, D. I. (2010). Appraising the ANT: Psychometric and theoretical considerations of the attention network test. Neuropsychology, 24(5), 637-651.

McConnell, M. M., \& Shore, D. I. (2011). Mixing measures: Testing an assumption of the Attention Network Test. Attention, Perception, \& Psychophysics, 73(4), 1096-1107.

Moruzzi, G., \& Magoun, H. W. (1949). Brain stem reticular formation and activation of the EEG. Electroencephalography and Clinical Neurophysiology, 1(4), 455-473. 
Mulckhuyse, M., \& Theeuwes, J. (2010). Unconscious attentional orienting to exogenous cues: A review of the literature. Acta Psychologica, 134(3), 299-309.

Muller, H. J., \& Rabbitt, P. M. (1989). Reflexive and voluntary orienting of visual attention: Time course of activation and resistance to interruption. Journal of Experimental Psychology: Human Perception and Performance, 15(2), 315-330.

Nakayama, K., \& Mackeben, M. (1989). Sustained and transient components of focal visual attention. Vision Research, 29(11), 1631-1647.

Niemi, P., \& Naatanen, R. (1981). Foreperiod and simple reaction time. Psychological Bulletin, 89(1), 133-162.

Nunnally, J. C. (1970). Introduction to psychological measurement. New York, NY: McGraw-Hill.

Olivers, C. N., \& Meeter, M. (2008). A boost and bounce theory of temporal attention. Psychological Review, 115(4), 836-863.

Petersen, S. E., \& Posner, M. I. (2012). The attention system of the human brain: 20 years after. Annual Review of Neuroscience, 35, 73-89.

Posner, M. I. (1980). Orienting of attention. Quarterly Journal of Experimental Psychology, 32, 3-26.

Posner, M. I. (2008). Measuring alertness. Annals of the New York Academy of Sciences, 1129, 193-199.

Posner, M. I., \& Boies, S. J. (1971). Components of attention. Psychological Review, 78(5), 391-408.

Posner, M. I., \& Cohen, Y. (1984). Components of visual orienting. In H. Bouma \& D. G. Bouwhuis (Eds.), Attention and performance X: Control of language processes (pp. 531-556). Hillsdale, NJ: Erlbaum.

Posner, M. I., \& Rothbart, M. K. (2007). Research on attention networks as a model for the integration of psychological science. Annual Review of Psychology, 58, 1-23.

Raz, A., \& Buhle, J. (2006). Typologies of attentional networks. Nature Reviews Neuroscience, 7(5), 367-379.

Redick, T. S., \& Engle, R. W. (2006). Working memory capacity and attention network test performance. Applied Cognitive Psychology, 20(5), 713-721.

Reeves, A., \& Sperling, G. (1986). Attention gating in short-term visual memory. Psychological Review, 93(2), 180-206.

Rosenberg, M. D., Finn, E. S., Scheinost, D., Papademetris, X., Shen, X., Constable, R. T., \& Chun, M. M. (2016). A neuromarker of sustained attention from whole-brain functional connectivity. Nature Neuroscience, 19(1), 165-171.

Rueda, M. R., Fan, J., McCandliss, B. D., Halparin, J. D., Gruber, D. B., Lercari, L. P., \& Posner, M. I. (2004). Development of attentional networks in childhood. Neuropsychologia, 42(8), 1029-1040.
Salthouse, T. A., \& Berish, D. E. (2005). Correlates of within-person (across-occasion) variability in reaction time. Neuropsychology, 19(1), 77-87.

Sara, S. J., \& Bouret, S. (2012). Orienting and reorienting: The locus coeruleus mediates cognition through arousal. Neuron, 76(1), $130-141$

Sawaki, R., \& Luck, S. J. (2010). Capture versus suppression of attention by salient singletons: Electrophysiological evidence for an automatic attend-to-me signal. Attention, Perception, \& Psychophysics, 72(6), 1455-1470.

Sergent, C., Wyart, V., Babo-Rebelo, M., Cohen, L., Naccache, L., \& Tallon-Baudry, C. (2013). Cueing attention after the stimulus is gone can retrospectively trigger conscious perception. Current Biology, 23(2), 150-155.

Smith, P. L., \& Ratcliff, R. (2009). An integrated theory of attention and decision making in visual signal detection. Psychological Review, $116(2), 283-317$.

Solomon, J. A. (2004). The effect of spatial cues on visual sensitivity. Vision Research, 44(12), 1209-1216.

Theeuwes, J. (2010). Top-down and bottom-up control of visual selection. Acta Psychologica, 135(2), 77-99.

Tsushima, Y., Sasaki, Y., \& Watanabe, T. (2006). Greater disruption due to failure of inhibitory control on an ambiguous distractor. Science, 314(5806), 1786-1788.

van Boxtel, J. J., Tsuchiya, N., \& Koch, C. (2010). Consciousness and attention: On sufficiency and necessity. Frontiers in Psychology, 1, 217.

Wang, Y. F., Jing, X. J., Liu, F., Li, M. L., Long, Z. L., Yan, J. H., \& Chen, H. F. (2015). Reliable attention network scores and mutually inhibited inter-network relationships revealed by mixed design and non-orthogonal method. Scientific Reports, 5, 10251.

Weinbach, N., \& Henik, A. (2012). Temporal orienting and alerting-The same or different? Frontiers in Psychology, 3, 236.

White, A. L., Lunau, R., \& Carrasco, M. (2014). The attentional effects of single cues and color singletons on visual sensitivity. Journal of Experimental Psychology: Human Perception and Performance, 40(2), 639-652.

Wright, R. D., \& Richard, C. M. (2000). Location cue validity affects inhibition of return of visual processing. Vision Research, 40(17), 2351-2358.

Yantis, S., \& Jonides, J. (1990). Abrupt visual onsets and selective attention: Voluntary versus automatic allocation. Journal of Experimental Psychology: Human Perception and Performance, 16(1), 121-134. 